\title{
Mapping soil texture classes using field texturing, particle size distribution and local knowledge by both conventional and geostatistical methods
}

\author{
T. OBERTHÜR ${ }^{a}, b, P . G O O V A E R T S^{c} \& A$. DOBERMAN ${ }^{b}$ \\ ${ }^{\mathrm{a}}$ University of Western Australia, Department of Geography, Nedlands, WA 6907, Australia, ${ }^{\mathrm{b}}$ International Rice Research Institute, \\ Soil and Water Sciences Division, PO Box 933, 1099 Manila, Philippines, and ${ }^{\mathrm{c}}$ The University of Michigan, Department of Civil \& \\ Environmental Engineering, EWRE Building, Ann Arbor, MI 48109-2125, USA
}

\begin{abstract}
Summary
We investigated the utility of three interpolation techniques that ignored descriptive 'soft' information and one that used it for mapping topsoil texture classes: re-coding of soil map units within Geographical Information Systems (GIS), Thiessen polygons, and classification of probability vectors estimated by ordinary indicator kriging and simple indicator kriging with local prior means. The results were compared with texture maps based on a classification of kriged maps of particle size distribution. The methods were applied to two distinct regions, which represent large areas in rain-fed rice ecosystems and irrigated rice ecosystems. The 'hard' databases for both areas contained soil information needed for mapping at regional scales (1:100000-1:150000). These data were complemented with 'soft' information derived from farmers and soil experts (Northeast Thailand) and soil maps (Nueva Ecija, Philippines).

Interpolated maps agreed with the texture map based on interpolation of particle size distribution, and field estimates of soil texture proved to be valuable surrogates for laboratory measurements of soil texture classes. The interpolation of categorical data such as soil texture classes allows for upgrading and increasing the resolution of maps in sparsely sampled regions by using simple field measurements.

Validation using independent test sets showed that indicator kriging with local prior means performed best in the rain-fed lands, whereas soft information did not improve the predictions in Nueva Ecija. Local knowledge in a formalized form was valuable in Northeast Thailand and the interpolated soil texture map for this area had an accuracy and resolution to support agronomic decisions at the village scale. The poor quality of the soil map and the fact that the gradually changing variability in young alluvial soils can be modelled with fewer data explained the lower accuracy of simple indicator kriging with local prior means in Nueva Ecija. Thiessen polygons performed well in the undulating rain-fed lands but were not as reliable as indicator kriging in the gradually changing irrigated lands.
\end{abstract}

\section{Introduction}

Soil maps and their accompanying reports are important sources of information on land resources. Their applicability, however, depends on survey and mapping scale, which is not appropriate for agronomic management decisions in many regions (see, for example, Oberthür et al., 1996). Furthermore, the efficacy of soil classification depends on the environment: Voltz \& Webster (1990) showed that classification performed satisfactorily where the soil changes abruptly, but not as well where the soil changes gradually. Also, conventional soil maps take no account of the within-class heterogeneity. Quantifying

Correspondence: T. Oberthür. E-mail: thomasob@gis.uwa.edu.au Received 27 October 1998; revised version accepted 31 March 1999 the uncertainty of the classification and increasing map resolution requires additional data, which can be used to produce more refined maps. Sampling and analysis of additional quantitative data are laborious and costly in heterogeneous rain-fed rice lands because survey effort increases with increasing intricacy in soil patterns (Bie \& Beckett, 1971). Alternatively, rapid field tests and existing soil survey reports provide valuable categorical information.

Categorical point information can be mapped using techniques such as Thiessen polygons. An alternative is to re-code map units using Geographical Information Systems with values from point sources to function as spatial carriers of information. Such simple approaches might not capture much of the variation within the classes, and the resulting maps may be of little value at larger scales. Kriging is now widely used 
for predicting continuous soil properties at unsampled locations. Unlike continuous variables, categorical variables cannot be estimated as mere linear combinations of neighbouring observations. Indicator geostatistics (Journel, 1983), based on a prior coding of information into indicator values, can handle categorical variables. Indicator (co-) kriging allows one to estimate the probability of occurrence of each class at unsampled locations (Goovaerts, 1994). The prediction calls for the derivation of a single class from the probability distribution, and classification algorithms such as the one developed by Soares (1992) might be used for this purpose.

Various techniques have recently been tested for their utility to improve the accuracy of geostatistical interpolation techniques at acceptable costs: adjusting kriging weights to the utility of available information (Heuvelink \& Bierkens, 1992) and kriging within strata (Boucneau etal., 1998). Alternatively, Journel (1986) employed indicator geostatistics to code jointly precise measurements and descriptive information, and Goovaerts \& Journel (1995) applied the principles to combine soil map information with laboratory data to model the spatial variation of continuous soil properties.

Information which can locally describe the natural resources in rice-growing environments includes remotely sensed data, topographic maps and knowledge of farmers and experts. This kind of descriptive information is referred to as 'soft' information because it relates indirectly to the primary attribute, which is defined as 'hard' information and measured on either a categorical or continuous scale. Indigenous soil knowledge has been much studied by anthropologists (e.g. Sandor \& Furbee, 1996), but few soil scientists and agronomists have used this knowledge for quantitative resource assessment (e.g. Arrouays, 1987), mainly because the integration of soft and hard information posed difficulties. We know of no study of the utility of local knowledge for improving spatial interpolations.

Most comparative studies of interpolation methods are concerned with the prediction of quantitative soil properties (Brus etal., 1996; Voltz \& Webster, 1990; Bárdossy \& Lehmann, 1998). Few investigators have studied categorical soil information: Bregt etal. (1992) compared Thiessen polygons with interpretation of representative profiles and subsequent re-coding of soil map units. Re-coding of a 1:200000 soil map proved to be the most reliable approach. Finke etal. (1996) studied the utility of representative soil profile information for characterizing spatial patterns of soil properties related to water and solute transport. The results discouraged the use of this type of information. Leenhardt et al. (1994) investigated the efficacy of soil map classification for predicting soil physical and hydrological properties at different scales. In comparison with information provided by simple and stratified random sampling, information from soil profiles served as an accurate predictor at large scales
$(<1: 25000)$ but was less effective at smaller scales $(1: 100000)$.

Soil texture classes are categorical variables, whereas the particle size fractions they summarize are continuous. How might hand-texturing of soil in the field compare with continuous data acquired in the laboratory as the basis for interpolation? Moreover, how might the integration of soft information from farmers and hard information from soil surveys improve the prediction of soil properties? To answer these questions we compared the performances of three techniques for mapping field-estimated topsoil texture classes (Thiessen polygons, re-coding of map unit information and indicator kriging) that ignore soft information with indicator kriging with local prior means that includes soft information. Results were also compared with texture classes derived by classifying interpolated maps of soil particle size distribution. The study was done in two disparate regions with sparse data, which are representative for large areas in rain-fed and irrigated rice-growing land.

\section{Case study 1: The young, alluvial plain in the Central Luzon Basin, Philippines}

Non-terraced alluvial plains are much used for lowland rice in South and Southeast Asia, and our study area of 19176 ha is part of such a plain in the province of Nueva Ecija, about $160 \mathrm{~km}$ north of Manila (Figure 1). The project area lies in a meandering flood plain, where the original geological sedimentation patterns have been reworked along the Talavera River. Elevation declines gradually from about $80 \mathrm{~m}$ above sea level in the northeast to $30 \mathrm{~m}$ above sea level in the southeast, and most of the area has been fully irrigated for more than 20 years. Two rice crops are grown per year.

The soil was classified and mapped during a reconnaissance soil survey in 1940 (Alicante etal., 1941), and the map $(1: 125000)$ is still a major source of soil information (Figure 1) in the Philippines. The soil was classified at the soil series level; within any one series the soil had similar natural drainage conditions, relief and type of parent material. Map units were almost exclusively delineated by qualitative soil profile descriptions at various sites and supported by few soil analyses; many soils mapped as silt loam have in fact a heavier texture (Oberthür etal., 1996). According to the US Soil Taxonomy (Soil Survey Staff, 1992) these soils would be mainly Vertisols (Ustic Endoaquerts and Ustic Epiaquerts), Alfisols and Inceptisols.

\section{Soil sampling}

Hard information. In 1993 we made a systematic soil survey, for which soil samples were collected at 341 locations on a regular $750 \mathrm{~m} \times 750 \mathrm{~m}$ grid and from 43 random locations. The average sampling intensity of this semi-detailed survey was one sample point per 50 ha, which supports mapping at scales of 


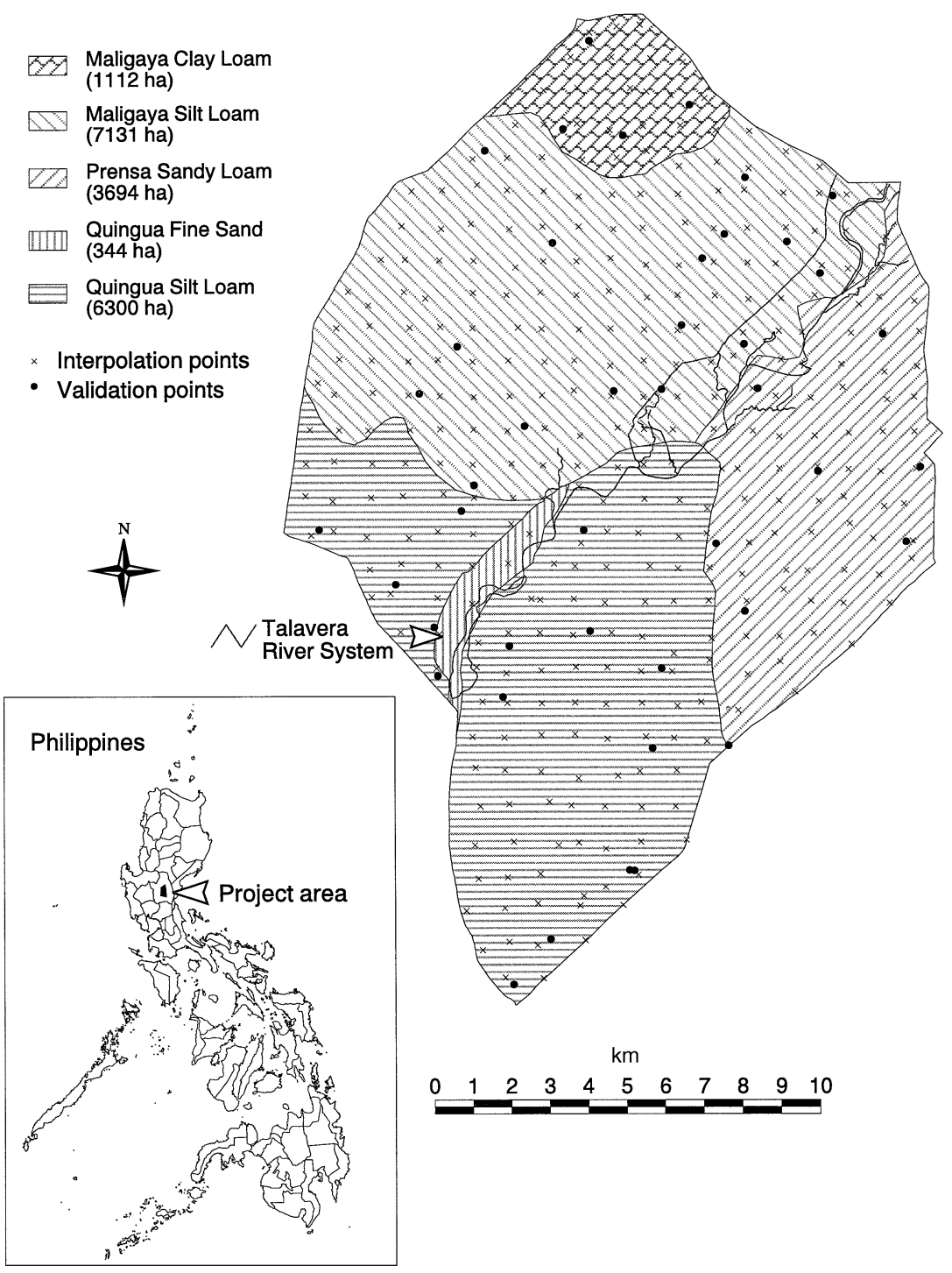

Figure 1 The Nueva Ecija project area with the sampling layout.

approximately 1:50 000 (Landon, 1991). Grid data were used for mapping, while the random points served as a validation set. Each location represented a small rice field. Soil samples were collected from 0 to $0.2 \mathrm{~m}$ depth (topsoil; soil monolith $0.2 \mathrm{~m} \times$ $0.2 \mathrm{~m} \times 0.2 \mathrm{~m}$ by spade) and from 0.2 to $0.4 \mathrm{~m}$ depth (subsoil; Dutch auger with $0.1 \mathrm{~m}$ diameter). Five soil samples were bulked, one from the centre of the field, and four within a 6-m radius around the centre of the field. The samples were air-dried and ground to pass through a 2-mm sieve. Soil texture was determined with the pipette method. Texture classes were estimated on auger borings in the field centre and from laboratory data using the US Department of Agriculture (Soil Survey Staff, 1951) soil texture classification.

Soft information. The only accessible source of soft information was the 1:125000 soil map of Alicante et al. (1941). An exhaustive soft data set was created by superimposing a $250 \mathrm{~m}$ $\times 250 \mathrm{~m}$ grid on the map and identifying the soil series at each of the 2973 grid nodes within a Geographical Information System (GIS).

\section{Case study 2: Old alluvio-colluvial, terraced land in Northeast Thailand}

Terraced, gently sloping lands are widespread throughout ricegrowing countries of Asia, and rain-fed rice is the dominant form of agriculture, although irrigation is applied where available. For this kind of land we studied a part of the Khorat Plateau (39025 ha), which is extensively used to grow rain-fed rice and lies within the basin of the lower Mae Nam Mun River. One of its tributaries, Lam Dom Yai, borders the study area in the southeast (Figure 2). Gently undulating land, 


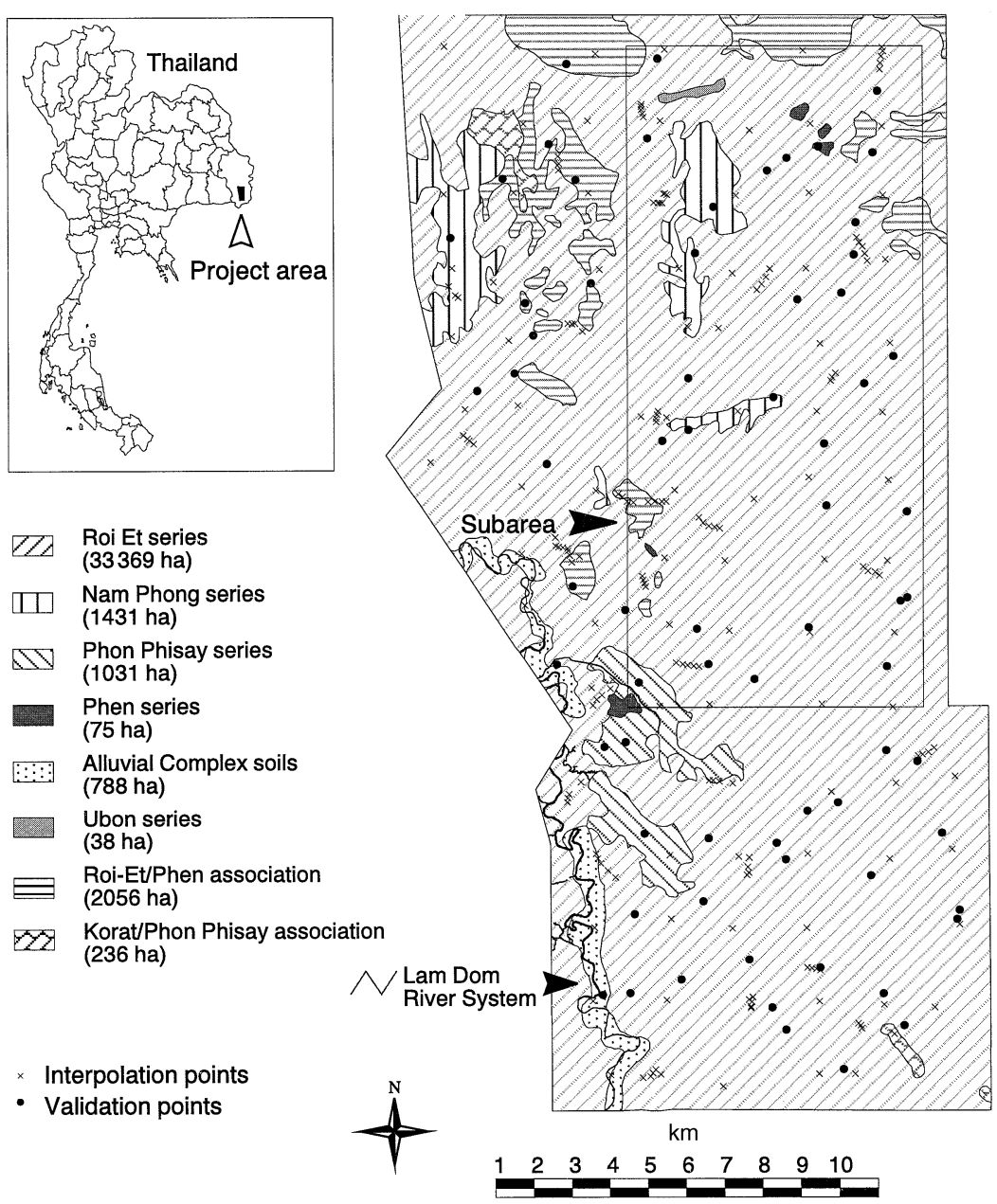

Figure 2 The Northeast Thailand project area with subarea and the sampling layout.

interspersed with minor non-flood and flood plains and some isolated hills, dominates the Khorat Plateau. The only available soil map (Figure 2), a semi-detailed reconnaissance map at 1:100000 scale (Changprai etal., 1971), is based on Moormann's soil development theory (Moormann etal., 1964), and soils in the project area include Aquults, Ustults, Psamments and Aquepts (Soil Survey Staff, 1992).

\section{Soil sampling}

Hard information. The sampling layout comprised three different sets to reveal soil heterogeneity over different spatial scales (Figure 2). A regular $2000 \mathrm{~m} \times 2000 \mathrm{~m}$ grid with 91 sample locations was drawn on a map, and fields nearest to these grid nodes were identified using aerial photographs and a Global Positioning System. To quantify short-range variation within microcatchments, 29 transects with a total number of 117 sampling sites were sampled in a stratified random design. Transect points and the grid points form the interpolation data set. A validation set was formed by specifying the coordinates of 70 additional fields in 14 strata using a random number generator. Strata were defined by dividing the region into 14 equal-sized regions. The samples support mapping at scales of approximately 1:100000 (Landon, 1991). The method of sampling was similar to that used in Nueva Ecija, except that the samples were taken from 0 to $0.15 \mathrm{~m}$ for the topsoil and $0.15-0.4 \mathrm{~m}$ for the subsoil. Samples were air-dried and ground to pass through a 2-mm sieve, and soil texture was determined using the hydrometer method. Soil texture classes were estimated in the field and determined in the laboratory using the US Department of Agriculture (Soil Survey Staff, 1951) soil texture classification.

Soft information. Farmers in Northeast Thailand characterize their fields according to toposequential position as low, medium and high land and integrate these features into riskavoiding cropping strategies. This knowledge was extracted in interviews and compiled for locations sampled in the aforementioned soil survey. The soil surveyor suggested that those fields with an upper sandy horizon, which is thicker than $1.5 \mathrm{~m}$, and fields frequently rejuvenated by alluvial sediments of the Lam Dom Yai River system should be included in the 
farmers' field classification (FFC) as additional classes. This classification is referred to as updated farmers' field classification (UFFC) and constitutes the soft information.

Exhaustive coverage of the soft information, which is required for simple indicator kriging with local prior means, was achieved in a 18000 -ha subarea of the original study area, for which scanned, pan-chromatic aerial photographs (1:4000) were acquired to facilitate a time and cost-efficient classification using the UFFC. Within a GIS, a $250 \mathrm{~m} \times 250 \mathrm{~m}$ grid was superimposed on the subarea to assign each grid node to one of the UFFC classes. Easily identifiable microcatchments dominate in Ubon Ratchathani, and the 2864 grid nodes of the subarea were allocated manually into UFFC classes using the scanned aerial photographs and an elevation map as visual aids. The procedure was validated in 1997 when locations of 100 randomly selected grid nodes were visited in the field and the classification accuracy of about $96 \%$ was considered as sufficient.

\section{Analytical methods for both study areas}

Processing of field soil texture classes prior to mapping

Large though they are, our data sets would not permit meaningful kriging if we were not to reduce the number of possible classes by merging some of those previously recognized. Thus, original topsoil texture classes as estimated in the field or given in the representative soil profile descriptions were aggregated into three major classes to obtain sufficient data for geostatistical modelling in each class. Relative to the average soil texture in the specific study area, these classes represent soils with light (class 1), medium (class 2) and heavy (class 3) texture.

In Nueva Ecija, class 1 comprises loamy sand, sandy loam, loam and silt loam, with only minor occurrences of loamy sand or sandy loam. Class 2 includes sandy clay loam, clay loam and silty clay loam. Clay and silty clay soils are in class 3 .

Northeast Thailand is characterized by coarse-textured soils. Consequently, class 1 in our definition represents sandy and loamy sand soils and class 2 sandy loam. All samples classified as loam and heavier are assigned to class 3 . Of course, the merging makes it easier for any interpolation method to hit the correct answer at any of the validation points; conversely, failure to do so is a more serious error than it would be if the number of classes were larger.

\section{Measures of within-unit variability}

The soil map units were analysed for within-unit variation by computing the complement of the relative variance (Webster \& Oliver, 1990), denoted here as $R V_{\mathrm{c}}$. The complement of the relative variance is a measure of the proportion of variance in the data explained by the classification. The total variance $\sigma_{\mathrm{T}}^{2}$ of an attribute $Z$ in the region formed by the mapping units can be given as (Leenhardt et al., 1994):
Table 1 Number of sites $(N)$ and the global proportion $(D)$ of field texture classes 1 (light), 2 (medium) and 3 (heavy) for Nueva Ecija and Northeast Thailand (total area and subarea)

\begin{tabular}{|c|c|c|c|c|c|c|}
\hline & & & \multicolumn{4}{|c|}{ Northeast Thailand } \\
\hline & \multicolumn{2}{|c|}{ Nueva Ecija } & \multicolumn{2}{|c|}{ Total area } & \multicolumn{2}{|c|}{ Subarea } \\
\hline & $N$ & $D$ & $N$ & $D$ & $N$ & $D$ \\
\hline Class 1 & 169 & 0.44 & 112 & 0.40 & 48 & 0.39 \\
\hline Class 2 & 109 & 0.28 & 103 & 0.37 & 45 & 0.36 \\
\hline Class 3 & 106 & 0.28 & 63 & 0.23 & 31 & 0.25 \\
\hline
\end{tabular}

$$
\sigma_{\mathrm{T}}^{2}=\sigma_{\mathrm{W}}^{2}=\sigma_{\mathrm{B}}^{2}
$$

where $\sigma_{\mathrm{B}}^{2}$ is the variation among the mapping unit means (between-unit variance) and $\sigma_{\mathrm{W}}^{2}$ is the variation occurring within each unit on average (within-unit variance). One-way analysis of variance was used to compute $s_{\mathrm{T}}^{2}, s_{\mathrm{B}}^{2}$ and $s_{\mathrm{W}}^{2}$, which estimate $\sigma_{\mathrm{T}}^{2}, \sigma_{\mathrm{B}}^{2}$ and $\sigma_{\mathrm{W}}^{2}$, respectively. The $R V_{\mathrm{c}}$ is then calculated as

$$
R V_{\mathrm{c}}=1-\left(\frac{s_{\mathrm{W}}^{2}}{s_{\mathrm{T}}^{2}}\right)
$$

Four mapping units of the soil map of Nueva Ecija (Maligaya Silt Loam, Maligaya Clay Loam, Prensa Sandy Loam and Quingua Silt Loam) and four mapping units of the soil map of Ubon Ratchathani (Roi Et, Roi Et/Phen, Phon Pisay, Nam Phong) were analysed for within-unit variation.

\section{Mapping of field texture classes}

Topsoil texture maps based on field texturing were generated by two conventional (see SM and TP below) and two geostatistical methods (see IK and SIK below) and compared with one another and with a map based on particle size distribution (see OK below). All variograms were visually fitted to a distance of $7-8 \mathrm{~km}$, which permitted us to take into account extensive knowledge about the area as well as experience gained during work in the regions on different scales. This was particularly useful for Nueva Ecija, where sampling distances did not account for potential short-range variability. Search radii for kriging were restricted to $3 \mathrm{~km}$. In Northeast Thailand, maps were produced only for the subarea, yet the complete data set was used to compute variograms under the assumption of stationarity. Such an assumption agrees with the similarity of the proportion of texture classes for the total and the subarea (Table 1). All sites of the original area where both soft and hard information was available were used for the calibration of hard and soft information (see below). 
Fitted variogram models include pure nugget variation, and linear combinations of models containing pure nugget variation and an exponential model (single or double) or pure nugget variation and an exponential and a spherical model. Pure nugget variation is defined as (Webster \& Oliver, 1990)

$$
\gamma(h)=c_{0}\{1-\delta(h)\}
$$

where $\gamma(h)$ is the semivariance, $c_{0}$ is the nugget variance, $h$ is the lag distance, and $\delta(h)$ is a function that takes the value 1 when $h=0$ and zero otherwise. The function

$$
\gamma(h)=\left[\begin{array}{ll}
c\left\{\frac{3 h}{2 a}-\frac{1}{2}\left(\frac{h}{a}\right)^{3}\right\} & \text { if } h \leq a \\
c & \text { if } h>a
\end{array}\right.
$$

defines a single spherical model. The shape of the function is determined by the range $a$ and the sill $c$. Single exponential models are defined by the function

$$
\gamma(h)=c\left\{1-\exp \left(-\frac{h}{r}\right)\right\},
$$

where $r$ is a distance parameter. The effective range of this function is usually taken as $3 r$, a distance for which the semivariance is approximately $0.95 c$ (Webster \& Oliver, 1990). The ARC/INFO GIS software (ESRI, 1991) and GSLIB (Deutsch \& Journel, 1992) were used for mapping by the following methods.

SM: Re-coding and classification of soil map units. In this approach the digitized soil maps (Figures 1,2) were used as base maps. The map units were re-coded within a GIS to display the representative texture class of each unit, which is mainly based on field estimation and obtained from the accompanying soil survey report.

TP: Thiessen polygons method. Thiessen polygons allow one to delineate the polygon of influence of each sampling point such that any location in the polygon is closer to the original sample point than to any other sampling points. Thiessen polygons were applied to field estimates of soil texture classes.

OK: Ordinary kriging. Maps of sand (Northeast Thailand) and clay (Nueva Ecija) content were produced from ordinary kriging estimates of laboratory data. Texture classes at each site were then obtained using the following classification.

Nueva Ecija: Class 1 clay $<35 \%$

Class 2 clay $35-50 \%$

Class 3 clay $>50 \%$

Northeast Thailand: Class 1 sand $>70 \%$

Class 2 sand $60-70 \%$

Class 3 sand $<60 \%$.
We acknowledge that this classification generates classes that are not exactly identical to the classes produced by grouping of field texture classes as described above. This modification may explain some of the differences between the final maps.

IK: Indicator kriging of soil texture classes, ignoring soft information. Indicator kriging was used to estimate, from soil texture classes determined in the field with the finger test, the probability of occurrence of each class at unsampled locations. The final categorical maps were obtained by applying Soares's algorithm (Soares, 1992) to the vector of local probabilities (see below).

SIK: Indicator kriging with local prior means, using soft information. Indicator kriging with local prior means was used to estimate, from hard (field estimations of soil texture classes) and soft information (the soil map in Nueva Ecija; knowledge of farmers and experts in Ubon Ratchathani), the probability of occurrence of each class at unsampled locations. Class maps were obtained using Soares's algorithm.

\section{The indicator approach}

Suppose $\left\{s_{k}, k=1,2, \ldots, K\right\}$ is a set of $K$ mutually exclusive soil texture classes with $n_{1}$ observations $\left\{s\left(\mathbf{u}_{\alpha}\right), \alpha=1,2, \ldots\right.$, $\left.n_{1}\right\}$ that are considered as precise measurements (hard information) of the class $s_{k}$ prevailing at $\mathbf{u}_{\alpha}$. Additional (soft) information that is available at all locations $\mathbf{u} \in A$ and relates indirectly to the hard data is provided. The soft information takes the form of a class $\nu_{l}$ out of a set of $L$ mutually exclusive classes, such as classes of elevation or land suitability. Using both hard and soft information one aims to estimate the probability for each soil texture class $s_{k}$ to prevail at any unsampled location $\mathbf{u}$ :

$$
\begin{gathered}
p\left\{\mathbf{u} ; s_{k} \mid\left(n_{1}+n_{2}\right)\right\}=\operatorname{Prob}\left\{S(\mathbf{u})=s_{k} \mid\left(n_{1}+n_{2}\right)\right\} \\
k=1,2, \ldots, K
\end{gathered}
$$

where the uncertainty about the unknown class at $\mathbf{u}, s(\mathbf{u})$, is modelled by the categorical random variable $S(\mathbf{u})$. The notation $\mathrm{I}\left(n_{1}+n_{2}\right)$ expresses the conditioning to the $n_{1}$ hard data $\left\{s\left(\mathbf{u}_{\alpha}\right), \alpha=1,2, \ldots, n_{1}\right\}$ and the $n_{2}$ soft data $\left\{\nu\left(\mathbf{u}_{\alpha}{ }_{\alpha}\right), \alpha=1\right.$, $\left.2, \ldots, n_{2}\right\}$ retained in the neighbourhood of $\mathbf{u}$. The conditional probability defined in Equation(6) can be interpreted as the conditional expectation of the indicator random function $I\left(\mathbf{u} ; s_{k}\right)$ given the information $\left(n_{1}+n_{2}\right)$. Thus, an estimate for $p\left\{\mathbf{u} ; s_{k} \mid\left(n_{1}+n_{2}\right)\right\}$ is provided by (co-)kriging $I\left(\mathbf{u} ; s_{k}\right)$ using the neighbouring indicator transforms of hard and soft data. The indicator co-kriging (CoIK) algorithm, which uses indicator data related to any class, is theoretically better because it accounts for transition probabilities between classes. Its implementation, however, requires joint modelling of $K(K+1) / 2$ cross indicator variograms and solving large and 
possibly unstable co-kriging systems. Moreover, Goovaerts (1994) showed that CoIK does not necessarily provide more accurate results than the straightforward indicator kriging, which involves modelling only the $K$ auto indicator variograms $\gamma_{\mathrm{I}}\left(\mathbf{h} ; s_{k}\right)$. We decided to use indicator kriging instead of cokriging because the latter approach is rarely implemented in practice.

\section{The indicator coding of information}

Joint processing of hard and soft information using indicator algorithms depends on preliminary coding of the data into local prior probabilities of type:

$$
\operatorname{Prob}\left\{S(\mathbf{u})=s_{k} \mid \text { local information at } \mathbf{u}\right\} k=1,2, \ldots, K .
$$

The probability defined in Equation(6) can be seen as the result of the updating of the local prior probability of Equation(7), and is referred to as a posterior probability. If there is no uncertainty about the soil texture class prevailing at a sampled location $\mathbf{u}_{\alpha}$ then the prior probability is 1 for the class recorded at $\mathbf{u}_{\alpha}$ and 0 for the $(K-1)$ other classes. At sampled locations, the local prior probabilities are binary (hard) indicator data defined as

$$
i\left(\mathbf{u}_{\alpha} ; s_{k}\right)=\left\{\begin{array}{l}
1 \text { if } s\left(\mathbf{u}_{\alpha}\right)=s_{k} \\
0 \text { otherwise }
\end{array} k=1,2, \ldots, K .\right.
$$

Where hard information is absent, the local prior probabilities are identified as the conditional frequencies $F^{*}\left(s_{k} \mid \nu_{l}\right)$ corresponding to the class $\nu_{l}$ prevailing at $\mathbf{u}$ :

$$
\begin{aligned}
y\left(\mathbf{u} ; s_{k}\right)= & F^{*}\left(s_{k} \mid \nu_{l}\right) \\
= & \frac{\sum_{\alpha=1}^{n} i\left(\mathbf{u}_{\alpha} ; s_{k}\right) i\left(\mathbf{u}_{\alpha} ; \nu_{l}\right)}{\sum_{\alpha=1}^{n} i\left(\mathbf{u}_{\alpha} ; \nu_{l}\right)} k=1,2, \ldots, K,
\end{aligned}
$$

where $i\left(\mathbf{u}_{\alpha} ; \nu_{l}\right)$ is an indicator of occurrence of class $\nu_{l}$, i.e. $i\left(\mathbf{u}_{\alpha} ; \nu_{l}\right)=1$ if $\nu\left(\mathbf{u}_{\alpha}\right)=\nu_{l}$ and zero otherwise. Whereas the binary (hard) indicator data $i\left(\mathbf{u}_{\alpha} ; s_{k}\right)$ take the values 0 or 1 , the soft data $y\left(\mathbf{u} ; s_{k}\right)$ have values between 0 and 1 because the attribute $\nu$ provides imprecise information about whether a particular class $s_{k}$ prevails at $\mathbf{u}$. The conditional frequencies $F^{*}\left(s_{k} \mid \nu_{l}\right)$ can be derived by calibration from those locations where both hard and soft data are known (Goovaerts, 1997, p. 329). The ability of the soft information to separate the two cases $i\left(\mathbf{u} ; s_{k}\right)=1$ and $i\left(\mathbf{u} ; s_{k}\right)=0$, that is to detect the presence of a specific class $s_{k}$, can be assessed from the calibration data using the following coefficient:

$$
\hat{B}\left(s_{k}\right)=\hat{m}^{(1)}\left(s_{k}\right)-\hat{m}^{(0)}\left(s_{k}\right) \in[-1,1],
$$

where $\hat{m}^{(1)}\left(s_{k}\right)$ is the arithmetic average of soft indicator data $y\left(\mathbf{u}_{\alpha} ; s_{k}\right)$ at locations where $i\left(\mathbf{u}_{\alpha} ; s_{k}\right)=1$ :

$$
\begin{aligned}
\hat{m}^{(1)}\left(s_{k}\right) & =\frac{1}{\sum_{\alpha=1}^{n} i\left(\mathbf{u}_{\alpha} ; s_{k}\right)} \\
& \sum_{\alpha=1}^{n} y\left(\mathbf{u}_{\alpha} ; s_{k}\right) i\left(\mathbf{u}_{\alpha} ; s_{k}\right) .
\end{aligned}
$$

Conversely, $\hat{m}^{(0)}\left(s_{k}\right)$ is the arithmetic average of the soft indicator data $y\left(\mathbf{u}_{\alpha} ; s_{k}\right)$ where $i\left(\mathbf{u}_{\alpha} ; s_{k}\right)=0$ :

$$
\begin{array}{r}
\hat{m}^{(1)}\left(s_{k}\right)=\frac{1}{\sum_{\alpha=1}^{n}\left\{1-i\left(\mathbf{u}_{\alpha} ; s_{k}\right)\right\}} \\
\sum_{\alpha=1}^{n} y\left(\mathbf{u}_{\alpha} ; s_{k}\right)\left\{1-i\left(\mathbf{u}_{\alpha} ; s_{k}\right)\right\} .
\end{array}
$$

Soft information $y$ exactly predicts that class $s_{k}$ prevails at $\mathbf{u}_{\alpha}$ if $\hat{m}^{(1)}\left(s_{k}\right)=1$ and conversely, exactly predicts that the class $s_{k}$ is absent at $\mathbf{u}_{\alpha}$ if $\hat{m}^{(0)}\left(s_{k}\right)=0$. The greater the coefficient $\hat{B}\left(s_{k}\right)$, the more information is provided by the soft data (Goovaerts, 1997, pp. 314-317).

\section{Indicator kriging with local prior means (SIK)}

Several indicator algorithms (e.g. indicator kriging with local prior means, co-located indicator co-kriging, full indicator cokriging) can be used to estimate each of the $K$ posterior probabilities $p\left\{\mathbf{u} ; s_{k} \mid\left(n_{1}+n_{2}\right)\right\}$ defined in Equation(6) as a linear combination of neighbouring hard and soft indicator data (Goovaerts \& Journel, 1995). We used simple indicator kriging with local prior means because it is easy to implement and avoids joint modelling of $K(K+1) / 2$ indicator (cross-) variograms. Using SIK, the global proportion of the class $s_{k}$, i.e. $p_{k}^{*}$, with $\sum_{k=1}^{K} p_{k}^{*}=1$, in the simple indicator kriging (sIK) estimator

$$
\begin{aligned}
& {\left[p\left\{\mathbf{u} ; s_{k} \mid\left(n_{1}\right)\right\}\right]_{\mathrm{sIK}}^{*}=\left\{i\left(\mathbf{u} ; s_{k}\right)\right\}_{\mathrm{sIK}}^{*}} \\
& =p_{k}^{*}+\sum_{\alpha=1}^{n} \nu_{\alpha}^{\mathrm{sIK}}\left(\mathbf{u} ; s_{k}\right) \cdot\left\{i\left(\mathbf{u}_{a} ; s_{k}\right)-p_{k}^{*}\right\}
\end{aligned}
$$

is replaced by the local soft prior probabilities $y\left(\mathbf{u} ; s_{k}\right)$ and $y\left(\mathbf{u}_{\alpha} ; s_{k}\right)$ to account for the soft information available at locations $\mathbf{u}$ and $\mathbf{u}_{\alpha}$. The term $\nu_{\alpha}^{\text {sIK }}\left(\mathbf{u} ; s_{k}\right)$ denotes the weight assigned to datum $i\left(\mathbf{u}_{\alpha} ; s_{k}\right)$. The estimate in Equation (13) is then rewritten:

$$
\begin{gathered}
{\left[p\left\{\mathbf{u} ; s_{k} \mid\left(n_{1}+1\right)\right\}\right]_{\mathrm{SIK}}^{*}=} \\
\left\{y\left(\mathbf{u} ; s_{k}\right)\right\}+\sum_{\alpha=1}^{n_{l}} \lambda_{\alpha}^{\mathrm{sIK}}\left(\mathbf{u} ; s_{k}\right)\left\{i\left(\mathbf{u}_{\alpha} ; s_{k}\right)-y\left(\mathbf{u}_{\alpha} ; s_{k}\right)\right\} .
\end{gathered}
$$

The weights are $\lambda_{\alpha}^{\mathrm{sIK}}\left(\mathbf{u} ; s_{k}\right)$ obtained by solving a simple indicator kriging system: 


$$
\begin{array}{r}
\sum_{\beta=1}^{n_{1}} \lambda_{\beta}^{\mathrm{sIK}}\left(\mathbf{u}_{\alpha} ; s_{k}\right) C_{R}\left(\mathbf{u}_{\alpha}-\mathbf{u}_{\beta} ; s_{k}\right)= \\
\left(\mathbf{u}_{\alpha}-\mathbf{u} ; s_{k}\right) \forall \alpha=1 \text { to } n_{1},
\end{array}
$$

where $C_{R}\left(\mathbf{h} ; s_{k}\right)$ is the covariance of the residual random function $R\left(\mathbf{u} ; s_{k}\right)=I\left(\mathbf{u} ; s_{k}\right)-y\left(\mathbf{u} ; s_{k}\right)$. At each location $\mathbf{u}$, the $K$ estimated probabilities, $\left[p\left\{\mathbf{u} ; s_{k} \mid\left(n_{1}+1\right)\right\}\right]^{*}$, must be valued within $[0,1]$ and must sum to 1 :

$$
\begin{gathered}
\left.p\left\{\mathbf{u} ; s_{k} \mid\left(n_{1}+1\right)\right\}\right] * \in[0,1] k=1,2, \ldots, K, \\
\sum_{k=1}^{K}\left[p\left\{\mathbf{u} ; s_{k} \mid\left(n_{1}+1\right)\right\}\right] *=1 .
\end{gathered}
$$

The first condition is not necessarily satisfied because kriging weights can be negative and therefore the kriging estimate is a non-convex linear combination of the conditioning data. Following Deutsch \& Journel (1992, p.72), the first constraint of Equation(16) is met by resetting the faulty probabilities to the nearest bound, 0 or 1 . The $K$ estimates $\left[p\left\{\mathbf{u} ; s_{k} \mid\left(n_{1}+1\right)\right\}\right]^{*} \in[0,1]$ are then divided by the sum $\sum_{k=1}^{K}\left[p\left\{\mathbf{u} ; s_{k} \mid\left(n_{1}+1\right)\right\}\right]^{*}$ to meet the second condition of Equation (17).

If the soft data do not allow a significant differentiation of $s$ values then the $L$ prior probabilities $F^{*}\left(s_{k} \mid \nu_{l}\right)$ would be similar to the global proportion $p_{k}^{*}$. The estimator then reverts to the simple indicator kriging estimator with constant indicator mean. The following steps were involved in simple indicator kriging with prior means (Goovaerts, 1997).

1 The proportion of fields where the estimated soil texture class is $s_{k}$ was first computed for each soft information class $\nu_{l}$. 2 The calibration results were used to convert the soft data into vectors of local prior probabilities $y\left(\mathbf{u} ; s_{k}\right)$, whereas fieldestimated texture classes were coded into hard indicator data $i\left(\mathbf{u}_{\alpha} ; s_{k}\right)$ using Equation (8).

3 The residual value $r\left(\mathbf{u}_{\alpha} ; s_{k}\right)$ was computed at all sampled locations $\mathbf{u}_{\alpha}$ by subtracting the soft indicator datum $y\left(\mathbf{u}_{\alpha} ; s_{k}\right)$ from the co-located hard indicator datum $i\left(\mathbf{u}_{\alpha} ; s_{k}\right)$. The experimental variogram of residuals was then computed and modelled.

4 The residual values were estimated at all unsampled grid nodes using simple kriging, and the posterior probability was obtained by adding the soft indicator datum $y\left(\mathbf{u} ; s_{k}\right)$ to the residual estimate $r^{*}\left(\mathbf{u} ; s_{k}\right)$.

\section{Indicator kriging $(I K)$}

If the soft information is ignored then the probability of Equation (6) is obtained as a linear combination of indicator data related only to the class $s_{k}$ being considered:

$$
\left[p\left\{\mathbf{u} ; s_{k} \mid\left(n_{1}\right)\right\}\right]_{\mathrm{IK}}^{*}=\sum_{\alpha=1}^{n_{1}} \lambda_{\alpha}^{\mathrm{IK}}\left(\mathbf{u} ; s_{k}\right) i\left(\mathbf{u}_{\alpha} ; s_{k}\right) .
$$

The weights are obtained by solving the following ordinary kriging system of $\left(n_{1}+1\right)$ equations:

$$
\left\{\begin{array}{c}
\sum_{\beta=1}^{n_{1}} \lambda_{\beta}^{\mathrm{IK}}\left(\mathbf{u} ; s_{k}\right) \gamma_{\mathrm{I}}\left(\mathbf{u}_{\alpha}-\mathbf{u}_{\beta} ; s_{k}\right)-\mu_{k}^{\mathrm{IK}}(\mathbf{u})=\gamma_{\mathrm{I}}\left(\mathbf{u}_{\alpha}-\mathbf{u} ; s_{k}\right) \\
\forall \alpha=1 \text { to } n_{1} \\
\sum_{\beta=1}^{n_{1}} \lambda_{\beta}^{\mathrm{IK}}\left(\mathbf{u} ; s_{k}\right)=1
\end{array}\right.
$$

where $\gamma_{\mathrm{I}}\left(\mathbf{h} ; s_{k}\right)$ is the indicator variogram, and $\mu_{k}^{\mathrm{IK}}(\mathbf{u})$ is the Lagrange parameter.

\section{Classifying the grid nodes}

Several classification criteria might be considered to allocate a node to a given class on the basis of the probability vector. Intuitively, one would tend to allocate each node $\mathbf{u}$ to the class associated with the largest local probability of occurrence. This criterion results in classifying most of the nodes into the most frequent classes since the local probability is likely to be larger if the corresponding proportion $p_{k}^{*}$ is already large. Such a classification is inadequate if one aims to reproduce the sample proportions, which are deemed representative of the entire study area (assumption of stationarity).

Soares (1992) developed a classification algorithm that preferentially allocates nodes to the class with the largest local probability of occurrence under the constraint of reproduction of the global proportions. The classification algorithm is dynamic in the sense that the allocation of a node can change as the classification progresses. The classification of $N$ grid nodes proceeds as follows (Goovaerts, 1997, p. 356).

1 For each class $s_{k}$ the $N$ grid nodes are ranked according to decreasing conditional probabilities $\left[p\left\{\mathbf{u} ; s_{k} \mid(n)\right\}\right]^{*}$.

2 The $n_{k}$ nodes $\left(n_{k}=N p_{k}^{*}\right)$ with the largest conditional probabilities $\left[p\left\{\mathbf{u} ; s_{k} \mid(n)\right\}\right]^{*}$ are allocated to the class $s_{k}$.

3 If a location $\mathbf{u}$ is allocated to two or more classes, e.g. $s_{k}$ and $s_{k}$, it is assigned to the class $s_{k}$ with the largest conditional probability of occurrence, i.e. where $\left[p\left\{\mathbf{u} ; s_{k} \mid(n)\right\}\right]^{*}>\left[p\left\{\mathbf{u} ; s_{k}\right.\right.$, $(n)\}]^{*}$. The $\left(n_{k},+1\right)$ th node with the largest conditional probability $\left[p\left\{\mathbf{u} ; s_{k}, \mid(n)\right\}\right]^{*}$ is allocated to class $s_{k}$, so that the global proportion $p_{k}^{*}$ is reproduced. The procedure is repeated until each grid node belongs to a single class.

\section{Validation of the mapping approaches}

The validation set was compared with the outcome of the classifications, and the results were arranged into an error 
Table 2 Example error matrix for $K$ classes and $N$ elements. Values in the main diagonal, here shown in bold for clarity, represent the number of elements of the interpolated and classified map that are classified correctly. Off-diagonal values indicate errors of omission and commission

\begin{tabular}{|c|c|c|c|c|c|c|}
\hline \multirow{2}{*}{$\begin{array}{l}\text { Interpolated and } \\
\text { classified map }\end{array}$} & \multirow[b]{2}{*}{1} & \multirow[b]{2}{*}{2} & \multicolumn{2}{|c|}{ Validation set } & \multirow[b]{2}{*}{$K$} & \multirow[b]{2}{*}{ Total } \\
\hline & & & $\ldots$ & $K-1$ & & \\
\hline 1 & $x_{11}$ & $x_{12}$ & $\ldots$ & $x_{1 K-1}$ & $x_{1 K}$ & $x_{1}$. \\
\hline 2 & $x_{21}$ & $x_{22}$ & $\ldots$ & $x_{2 K-1}$ & $x_{2 \mathrm{~K}}$ & $x_{2}$ \\
\hline . & $\cdot$ & $\cdot$ & . & $\cdot$ & . & . \\
\hline . & . & . & . & . & . & . \\
\hline$\cdot$ & $\cdot$ & $\cdot$ & . & $\cdot$ & $\cdot$ & . \\
\hline$K-1$ & $x_{K-11}$ & $x_{K-12}$ & $\ldots$ & $x_{K-1} K-1$ & $x_{K-1 K}$ & $x_{(K-1)}$ \\
\hline K & $x_{K 1}$ & $x_{K 2}$ & $\ldots$ & $x_{K K-1}$ & $x_{K K}$ & $x_{K}$ \\
\hline Total & $x_{\bullet 1}$ & $x_{\bullet 1}$ & $\ldots$ & $x \cdot(K-1)$ & $x_{\bullet K}$ & $N$ \\
\hline
\end{tabular}

matrix, which summarizes the overlap between estimated elements (pixels) and elements of the validation set. Table 2 gives an example for $N$ elements and $K$ classes. The term in the error matrix, denoted $x_{l k}$, represents the number of elements that are classified into class $l$ for the interpolated map and class $k$ for the reference map (validation set). Terms in the major diagonal (i.e. $l=k$ ) represent agreement between the two maps. The marginal row totals, denoted as $x_{l \cdot}$, are computed as

$$
x_{l \bullet}=\sum_{k=1}^{K} x_{l k} .
$$

The term $x_{\bullet_{k}}$ denotes the marginal column totals, which are calculated as

$$
x_{\bullet k}=\sum_{l=1}^{L} x_{l k} .
$$

Classification accuracy is then computed as the proportion of correctly classified elements (PCC) for the $k$ th class as

$$
\mathrm{PCC}_{k}=\frac{x_{k k}}{x_{\bullet k}}
$$

and globally as

$$
\mathrm{PCC}=\frac{\sum_{k=1}^{K} x_{k k}}{N} .
$$

The PCC parameter does not consider off-diagonal elements (errors of omission and commission) and the Kappa statistic (Cohen, 1960), here denoted with $A$, was therefore globally estimated according to Bishop etal. (1975) as

$$
A=\frac{N \sum_{k=1}^{K} x_{k k}-\sum_{k=1}^{K} x_{k \bullet} x_{\bullet k}}{N^{2}-\sum_{k=1}^{K} x_{k \bullet} x_{\bullet k}},
$$

and conditionally for each class $k$ by

$$
A_{k}=\frac{N x_{k k}-x_{k \bullet} x_{\bullet k}}{N x_{k \bullet}-x_{k \bullet} x_{\bullet k}}
$$

The estimates of Equations (24) and (25) are the proportion of agreement not due to chance and can range from -1 to 1 , where 1 represents full agreement between true and estimated classes.

The Kappa statistic does not account for the severeness of misclassification, yet a case of light soil texture wrongly allocated to medium soil texture is not as erroneous as light texture allocated to heavy texture. Therefore, Jolayemi's $r$ statistic (Jolayemi, 1990) was also computed as

$$
r=\sqrt{\frac{\chi^{2}}{(k-1) N}}
$$

where $\chi^{2}$ is the Pearson chi-squared statistic to test the independence for the observed configuration in the error matrix. The $r$ coefficient, $-1<r<1$, is a measure of agreement between the actual and predicted classes, which accounts for the size of error that is associated with misclassification (Foody, 1994).

\section{Results}

\section{Soil texture information from hard and soft data}

Nueva Ecija. Average clay and silt contents in the Nueva Ecija area were $42.6 \%$ and $37.8 \%$, respectively (Table 3). A quarter of the area has very heavy-textured soils with clay and silt content exceeding $52 \%$ and $43 \%$, respectively. Parts of the area are light to medium textured as shown by sand content greater than $28 \%$ in a quarter of the samples, mainly in the east 
Table 3 Descriptive statistics and the complement of the relative variance for the irrigated rice lands in Nueva Ecija (Philippines; 384 samples) and the rain-fed rice lands in Northeast Thailand (278 samples). Presented values are based on laboratory analysis of topsoil samples (0-20 cm for Nueva Ecija, $0-15 \mathrm{~cm}$ for Northeast Thailand). Mean laboratory values of particle size distribution are also given for sites allocated to the field-estimated texture classes 1 (light), 2 (medium) and 3 (heavy)

\begin{tabular}{|c|c|c|c|c|c|c|c|c|c|c|c|c|c|}
\hline & Mean & $\mathrm{SD}^{\mathrm{a}}$ & $\mathrm{CV}^{\mathrm{b}}$ & $\operatorname{Min}^{c}$ & $\mathrm{LQ}^{\mathrm{d}}$ & $\mathrm{Med}^{\mathrm{e}}$ & $U^{f}$ & $\operatorname{Max}^{\mathrm{g}}$ & $\mathrm{Sk}^{\mathrm{h}}$ & $R V_{\mathrm{c}}^{\mathrm{i}}$ & Mean $1^{\mathrm{j}}$ & Mean $2^{\mathrm{k}}$ & Mean $3^{1}$ \\
\hline \multicolumn{14}{|c|}{ Nueva Ecija } \\
\hline Clay $1 \%$ & 42.6 & 13.4 & 0.31 & 13.6 & 32.8 & 42.2 & 52.5 & 72.6 & 0.12 & 0.15 & 33.8 & 45.6 & 53.5 \\
\hline Silt $1 \%$ & 37.8 & 8.4 & 0.22 & 22.2 & 31.1 & 36.6 & 43.2 & 63.0 & 0.55 & 0.20 & 39.4 & 37.6 & 35.5 \\
\hline Sand $1 \%$ & 19.6 & 12.8 & 0.65 & 0.6 & 9.0 & 17.2 & 28.9 & 53.4 & 0.55 & 0.22 & 26.9 & 16.8 & 11.0 \\
\hline \multicolumn{14}{|c|}{ Northeast Thailand } \\
\hline Clay $1 \%$ & 9.4 & 7.2 & 0.77 & 1.0 & 5.3 & 7.2 & 10.4 & 48.4 & 2.60 & 0.01 & 6.0 & 8.9 & 16.1 \\
\hline Silt $1 \%$ & 20.5 & 10.5 & 0.51 & 0.1 & 12.1 & 18.2 & 27.9 & 54.1 & 0.77 & 0.05 & 14.3 & 22.8 & 27.9 \\
\hline Sand $1 \%$ & 70.1 & 15.0 & 0.21 & 23.3 & 60.9 & 73.1 & 82.2 & 92.5 & -0.96 & 0.05 & 79.8 & 68.2 & 55.8 \\
\hline
\end{tabular}

${ }^{\mathrm{a}}$ Standard deviation; ${ }^{\mathrm{b}}$ coefficient of variance; ${ }^{\mathrm{c}}$ minimum; ${ }^{\mathrm{d}}$ lower quartile, ${ }^{\mathrm{e}}$ median; ${ }^{\mathrm{f}}$ upper quartile; ${ }^{\mathrm{g}}$ maximum; ${ }^{\mathrm{h}}$ skewness; ${ }^{\mathrm{i}}$ complement of the relative variance for the 1:125000 (Nueva Ecija) and 1:100000 (Northeast Thailand) soil maps; ${ }^{\mathrm{j}}$ mean for field texture class 1 (light); ${ }^{\mathrm{k}}$ mean for field texture class 2 (medium); ${ }^{1}$ mean for field texture class 3 (heavy).

Table 4 Probability of occurrence of texture classes 1-3 conditionally to the presence of soft information (soil series, topographic position). The calibration sets include 384 observations for the Nueva Ecija study region and 278 observations for Northeast Thailand; the number of observations within each class is given in parentheses. The last column gives the $\hat{B}\left(s_{k}\right)$ coefficient that measures the ability of the soft information to predict each texture class

\begin{tabular}{|c|c|c|c|c|c|c|}
\hline Nueva Ecija & Soil Series $1^{\mathrm{a}}$ & Soil Series $2^{\mathrm{b}}$ & Soil Series $3^{\mathrm{c}}$ & Soil Series $4^{\mathrm{d}}$ & Soil Series $5^{\mathrm{e}}$ & $\hat{B}\left(s_{k}\right)$ \\
\hline Class 1 & $0.4(2)$ & $0.64(46)$ & $0.40(55)$ & $0.38(55)$ & $0.54(13)$ & 0.04 \\
\hline Class 2 & $0.6(3)$ & $0.21(15)$ & $0.22(31)$ & $0.32(47)$ & $0.46(11)$ & 0.03 \\
\hline Class 3 & $0.0(0)$ & $0.15(11)$ & $0.38(52)$ & $0.30(43)$ & $0.0(0)$ & 0.06 \\
\hline Northeast Thailand & Position $1^{\mathrm{f}}$ & Position $2^{\mathrm{g}}$ & Position $3^{\mathrm{h}}$ & Position $4^{\mathrm{i}}$ & Position $5^{\mathrm{j}}$ & $\hat{B}\left(s_{k}\right)$ \\
\hline Class 1 & $0.15(11)$ & $0.39(41)$ & $0.56(37)$ & $0.91(21)$ & $0.11(1)$ & 0.18 \\
\hline Class 2 & $0.31(22)$ & $0.46(50)$ & $0.39(26)$ & $0.09(3)$ & $0.33(3)$ & 0.04 \\
\hline Class 3 & $0.54(38)$ & $0.15(17)$ & $0.05(3)$ & $0.0(0)$ & $0.56(5)$ & 0.39 \\
\hline
\end{tabular}

${ }^{\mathrm{a}}$ Quingua Fine Sand; ${ }_{\mathrm{b}}^{\mathrm{b}}$ Prensa Sandy Loam; ${ }^{\mathrm{c}}$ Quingua Silt Loam; ${ }^{\mathrm{d}}$ Maligaya Silt Loam; ${ }^{\mathrm{e}}$ Maligaya Clay Loam; ${ }^{\mathrm{f}}$ Low; ${ }^{\mathrm{g}}$ Medium; ${ }^{\mathrm{h}} \mathrm{High} ;{ }^{\mathrm{i}}$ Deep sands; ${ }^{\mathrm{j}}$ Alluvial sites.

and along the Talavera River. The existing soil map could explain only $15-22 \%$ of the variation in clay, silt and sand (Table 3).

Hand texture estimation resulted in 169 light, 109 medium and 106 heavy-textured soil samples (Table 1). The change in soil texture is due mainly to a change in clay and sand content. The clay content in the heavy class $(53.5 \%)$ is on average about 20\% larger than in the light class (33.8\%). Sand averages $26.9 \%$ in class 1 and $11 \%$ in class 3 . Average silt content differed little among all three classes (35.5-39.4\%).

Table 4 shows, for each soil series, the probabilities of occurrence of the three texture classes, which were derived from the calibration set of 384 observations. These prior probabilities $F^{*}\left(s_{k} \mid \nu_{l}\right)$ were used to derive the $\hat{B}\left(s_{k}\right)$ coefficients that measure the ability of the soft information to predict the presence of each texture class. The small coefficients indicate that the soft information has little ability to predict texture classes.

Northeast Thailand. Soil texture in the Northeast Thailand study area is coarser than in Nueva Ecija, and the three texture classes were different in sand, silt and clay contents (Table 3). Half of the samples have more than $70 \%$ sand and less than $8 \%$ clay. Average clay contents ranged from $6 \%$ in class 1 to $16.1 \%$ in class 3 . The average sand content ranged from $55.8 \%$ in class 3 to $79.8 \%$ in class 1 . The $R V_{\mathrm{c}}$ indicates that the soil map did not explain more than $5 \%$ of the variation in sand, silt and clay content. There are 112 samples in the light-textured class while 103 and 63 are in the medium and heavy-textured class, respectively (Table 1).

Differences between conditional probabilities are more pronounced for Northeast Thailand, and larger $\hat{B}\left(s_{k}\right)$ coeffi- 

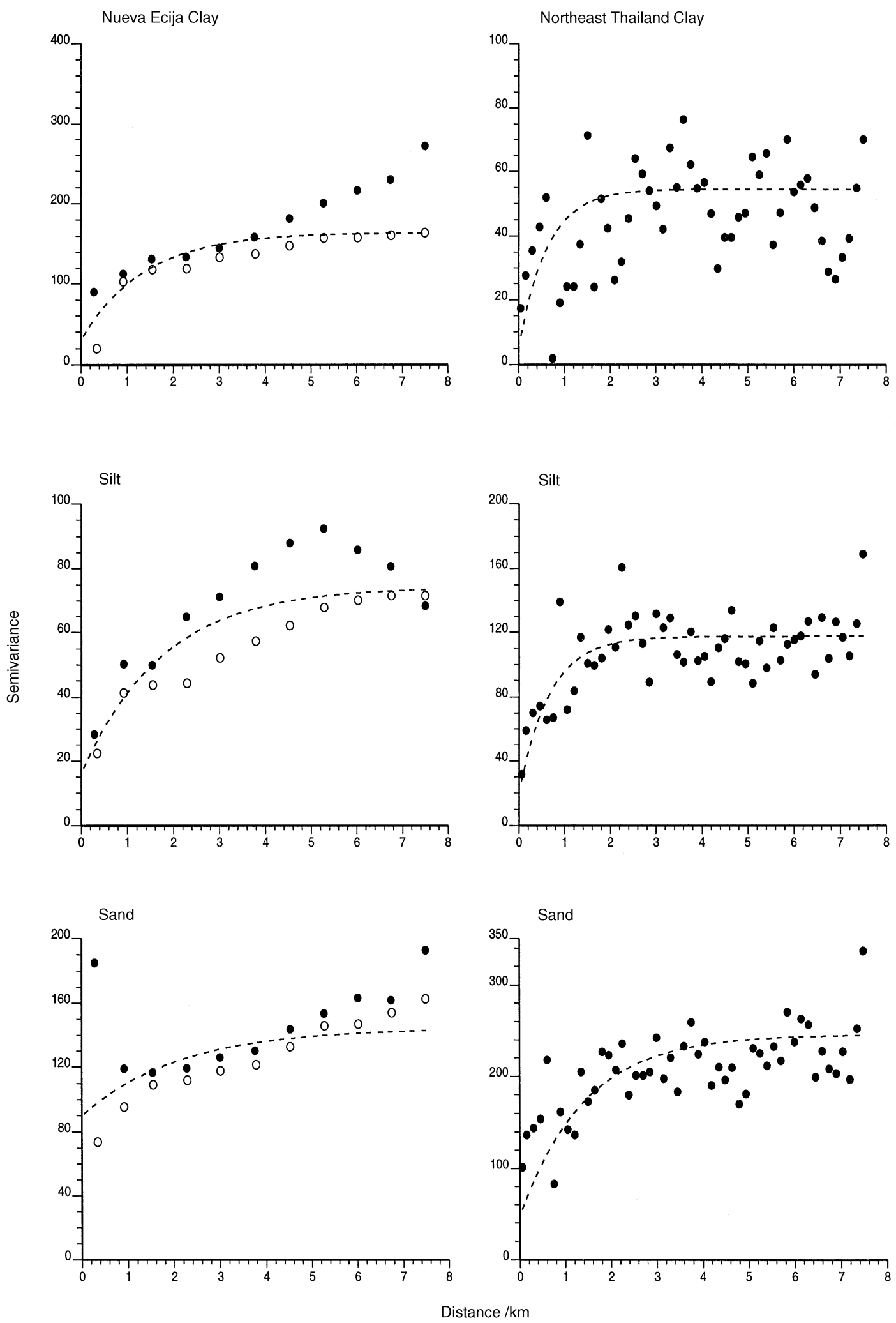

Figure 3 Experimental variograms and fitted models (dashed line) for soil particle size distribution in the two study areas. Variation is slightly anisotropic in Nueva Ecija; the directions shown are $30^{\circ}$ (open circles) and $120^{\circ}$ (filled circles) clockwise from north. 
cients (0.04-0.39) suggest better predictive power of topographic field positions (Table 4).

\section{Spatial correlation of soil texture}

Nueva Ecija. The semivariance for all particle size classes increased gradually after sharp initial increases over the first $2 \mathrm{~km}$, which indicates a notable amount of short-range variation (Figure 3).

All classes had a slightly anisotropic spatial structure, with clay and sand showing a trend in the approximately NW to SE direction $\left(120^{\circ}\right.$ clockwise from north) perpendicular to the flow direction of the Talavera River (Figures 1, 3). Spatial correlation is evident for distances up to $6 \mathrm{~km}$ for silt. The variograms for clay and sand indicate spatial correlation up to $5 \mathrm{~km}$ in direction $30^{\circ}$ (approximately NE). We fitted isotropic models to the experimental variograms because the anisotropy is not pronounced.

The indicator variograms for classes 1 and 3 reached a sill at about $7 \mathrm{~km}$, and for class 2 the variogram levels out at about $8 \mathrm{~km}$ (Figure 4). The relative nugget variance was larger for class 2 than for other ones (Table 5). Indicator variograms were modelled with a pronounced short-range structure to account for small inclusions with light and heavy texture, which were encountered during field work but not fully captured by the sampling design.

The variograms of residuals (Figure 5) have nuggets similar to the ones of the indicator variograms for texture classes (except class 3; Figure 4) because all prior probabilities are not very different (poor discriminatory power of the soil series); see the rows in Table 4. The fitted spherical models reach their sills at about $6 \mathrm{~km}$ and have nugget variances from 0.003 to 0.13 (Table 5).

Northeast Thailand. Unlike Nueva Ecija, most of the variation in clay, silt and sand content in Northeast Thailand occurred over distances of less than $2 \mathrm{~km}$, and no directional trends were observed (Figure 3). The exponential models fitted to the experimental variograms of clay and silt reach their sills effectively at about $2 \mathrm{~km}$ (distance parameters of $0.62 \mathrm{~km}$ and $0.667 \mathrm{~km}$, respectively), whereas for sand the effective range is about $4 \mathrm{~km}$ (distance parameter of $1.4 \mathrm{~km}$; Table 6, Figure 3 ).

The indicator variogram for class 1 has two nested structures with distance parameters of about $0.14 \mathrm{~km}$ and $2.04 \mathrm{~km}$. Soil texture class 2 appears as pure nugget. The experimental indicator variogram for class 3 was modelled using two exponential structures of effective ranges $1.4 \mathrm{~km}$ and $7.9 \mathrm{~km}$ (distance parameters of about $0.47 \mathrm{~km}$ and $2.6 \mathrm{~km}$; Table 6 , Figure 4).

Short-range variation, which was observed along the toposequential transects in the region, is reflected in the exponential models fitted to the residual variograms that reach their sills shortly before or at around $1 \mathrm{~km}$ (Figure 5). Unlike the indicator variograms, the residual variograms display only short-range structures and small nugget effects (except class 1; Table 6). The visual differences between variograms of residuals and indicator variograms, most notably for the medium texture class, emphasize the discriminatory power of the soft information (see rows in Table 4).

\section{Maps of soil texture classes}

Nueva Ecija. The map based on re-coding of representative profiles (Figure 6b) showed no agreement with any of the other texture maps because, on the basis of the soil series, most of the area was allocated to class 1 (Table 7). The TP map (Figure 6c), the OK map (Figure 7a), the IK map (Figure 7b) and the SIK map (Figure 7c) exhibit similar trends and reflect the distribution patterns of clay (Figure 6a): all classes are represented, the soil texture changes from heavy to light in the west-east direction and medium texture encloses heavy soil texture west of the centre. Another concentration of heavy texture can be seen in the south. All maps show a corridor with soil texture class 1 south of the centre.

Differences become apparent if the maps are examined more closely, however: the TP map is most fractured and appears unrealistically blocky. The indicator kriging map reveals much detail but is less patchy than the TP map. Most spatial consistency is seen in the maps generated using OK and SIK. Compared with IK, SIK classifies less land in the south as heavy textured, whereas more medium-textured soil is found in the north.

Northeast Thailand. Re-coding of the original soil map did not match the proportions of soil texture classes estimated from other maps because much land was assigned to class 2 (Table 7, Figure 8b). The Roi Et soil series (sandy loam; Figure 2) dominates the original soil map, and soil texture classes 1 and 3 are assigned only to marginal areas, although they seem to cover much more land in reality. Spatial patterns of texture classes in the map generated using OK (Figure 9a) expectedly mirror the patterns of the distribution of sand (Figure 8a). Thiessen polygons (Figure 8c) and IK (Figure 9b) are similar and show more heavy soil texture than the OK map, but Thiessen polygons do not realistically present information about short-range variation from transects, which the algorithm maps as elongated artefacts. The SIK map reveals a dendritic pattern and much spatial detail of soil texture class distribution. Texture classes change gradually from heavy to medium to light and follow the main topographic and drainage pattern. Larger coherent inclusions of light soil texture in the centre and of medium to heavy soil texture in the NW and SW suggest also that factors other than topography and drainage influence the spatial distribution of texture classes. 

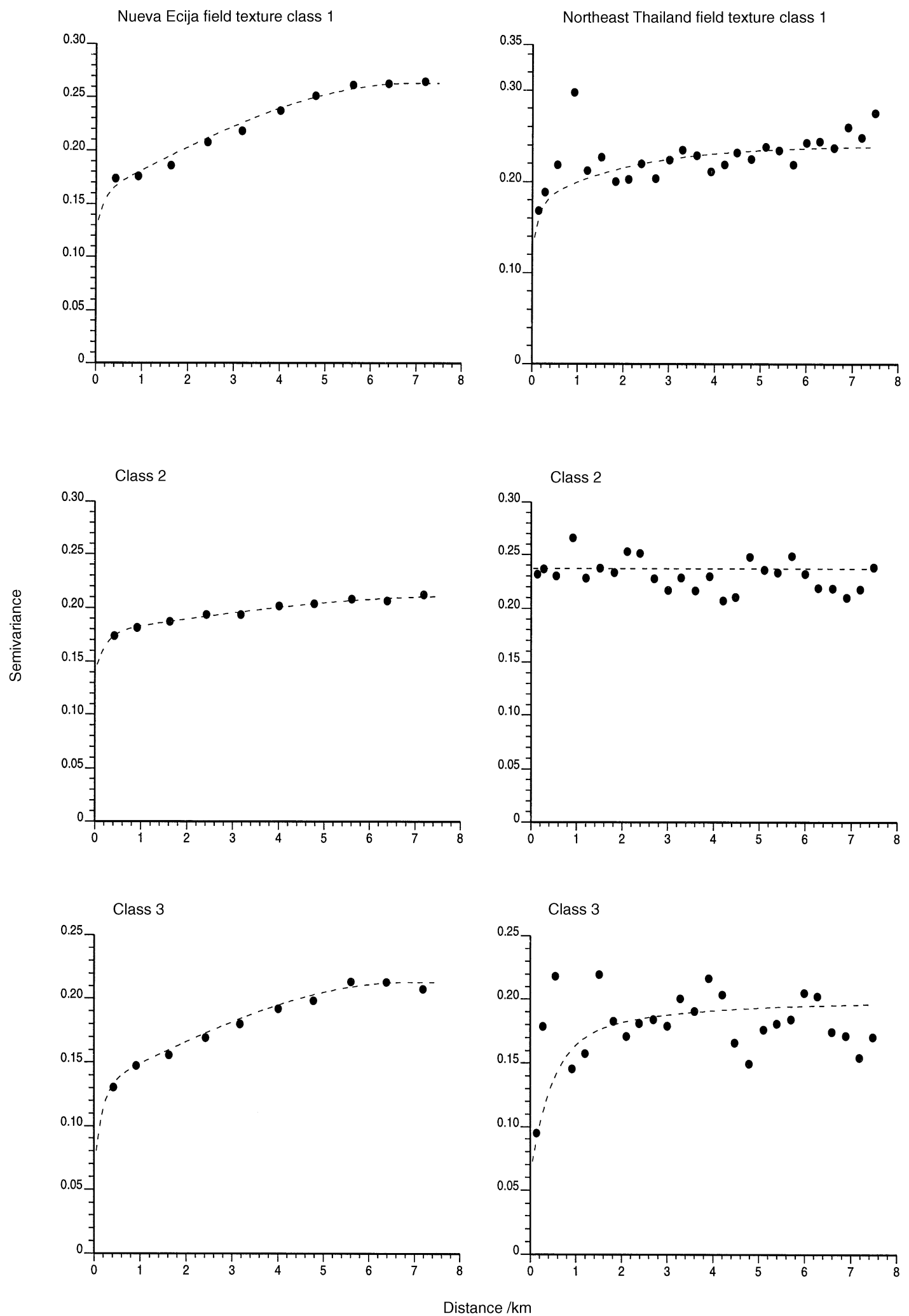

Figure 4 Experimental, omnidirectional indicator variograms with the fitted models (dashed line) for soil texture classes 1-3 in both areas. 
Table 5 Variogram parameters for Nueva Ecija (based on 384 samples)

\begin{tabular}{|c|c|c|c|c|c|c|c|c|c|}
\hline & $\mathrm{VM}_{1}{ }^{\mathrm{a}}$ & $c_{0}^{\mathrm{b}}$ & $c_{1}{ }^{\mathrm{c}}$ & $r_{1} / \mathrm{m}^{\mathrm{d}}$ & $a_{1} / \mathrm{m}^{\mathrm{e}}$ & $\mathrm{VM}_{2}{ }^{\mathrm{a}}$ & $c_{2}{ }^{\mathrm{f}}$ & $r_{2} / \mathrm{m}^{\mathrm{g}}$ & $a_{2} / \mathrm{m}^{\mathrm{h}}$ \\
\hline \multicolumn{10}{|c|}{ Variograms } \\
\hline Clay & $\exp$ & 30.1 & 164.4 & 1362 & - & - & - & - & - \\
\hline Silt & $\exp$ & 16.0 & 74.3 & 1752 & - & - & - & - & - \\
\hline Sand & $\exp$ & 90.0 & 144.5 & 2131 & - & - & - & - & - \\
\hline \multicolumn{10}{|c|}{ Indicator variograms } \\
\hline Class 1 & $\exp$ & 0.123 & 0.159 & 153 & - & $\mathrm{sph}$ & 0.263 & - & 6949 \\
\hline Class 2 & $\exp$ & 0.138 & 0.176 & 221 & - & $\mathrm{sph}$ & 0.209 & - & 7920 \\
\hline Class 3 & $\exp$ & 0.061 & 0.131 & 170 & - & sph & 0.212 & - & 6847 \\
\hline \multicolumn{10}{|c|}{ Variograms of residuals } \\
\hline Class 1 & $\mathrm{sph}$ & 0.130 & 0.270 & - & 5898 & - & - & - & - \\
\hline Class 2 & sph & 0.106 & 0.259 & - & 6540 & - & - & - & - \\
\hline Class 3 & sph & 0.003 & 0.028 & - & 6423 & - & - & - & - \\
\hline
\end{tabular}

${ }^{\mathrm{a}}$ Variogram models for the short-range $\left(\mathrm{VM}_{1}\right)$ and long-range structures $\left(\mathrm{VM}_{2}\right)$ : exp - exponential model, sph - spherical model; ${ }^{b}$ nugget variance; ${ }^{\mathrm{c}}$ sill of the short-range variance; ${ }^{\mathrm{d}}$ distance parameter of the short-range structure; ${ }^{\mathrm{e}}$ range parameter of the short-range structure; ${ }^{\mathrm{f}}$ sill of the long-range variance; ${ }^{g}$ distance parameter of the long-range structure; ${ }^{h}$ range parameter of the long-range structure.

\section{Map validation}

Nueva Ecija. Indicator kriging performed well when the interpolated maps were tested against the validation set (Table 8): the coefficient of the Kappa statistic was larger (0.28) than that of the SM map $(-0.06)$, the OK map (0.22) and the TP method (0.19). The conditional coefficients of the Kappa statistic confirm that IK performed well for soil texture classes 1 and 3 , but not for class 2 . Good results were also achieved with TP for class 3. However, Table 8 indicates that accounting for soft information scarcely increases the classification accuracy: the PCC (0.55 against 0.53$)$ and $A$ (0.28 against 0.27 ) are similar for IK and SIK. But indicator kriging with local prior means is the only method that produces good results for all classes. Also, SIK generates slightly less severe misclassifications than IK as indicated by the $r$ statistic $(0.20$ against 0.19$)$ but $\mathrm{OK}$ is less accurate in this context (0.13).

Northeast Thailand. Unlike in Nueva Ecija, the integration of soft information improved mapping in the Northeast Thailand subarea (Table 9): agreement between the validation set and estimated texture classes was closer for SIK (PCC $=0.66$, $A=0.37)$ than for IK $(\mathrm{PCC}=0.53, A=0.21)$, OK $(\mathrm{PCC}=0.50$, $A=0.06)$ or TP $(\mathrm{PCC}=0.55, A=0.27)$. The Kappa and PCC values suggest enhanced classification accuracy for SIK for heavy texture as already suggested by a large $\hat{B}\left(s_{k}\right)$ coefficient (Table 4), but only two validation sites were available in this class, and careful interpretation is required. Soft information reduces not only the proportion of wrongly classified land, but the large $r$ statistic also proves that misclassifications using SIK (0.30) are not as severe as those generated by the soil map method (0.06), indicator kriging (0.08), ordinary kriging $(0.07)$ or Thiessen polygons (0.13).

\section{Discussion for both environments}

Methodological aspects

Maps based on field texturing (i.e. SIK, IK and TP) resemble the OK map, and this confirms the finding of Hodgson et al. (1976) that sound estimation of soil texture in the field is practically feasible. Some of the deviations can be explained by the fact that the relation between field and laboratory soil texture classes can be considered only as an approximation (Kuntze etal., 1994). Also, grouping of US Department of Agriculture field texture classes generated three broader classes for interpolation, which were not identical to classes generated by applying thresholds to ordinary kriging maps of sand and clay. It may also be necessary to obtain additional field data where other soil factors bias the finger texture estimation, and we recommend intensive calibration with reference samples for both areas. Surveyors have to be cautious not to underestimate clay content for both regions in places where microaggregates of clay and iron oxides give the soil a sandy feel. In Northeast Thailand, clay contents are sometimes locally overestimated because of larger than average $\mathrm{CaCO}_{3}$ content in the soil.

If soft information was ignored, the simple Thiessen polygons algorithm proved to be as efficient as more complex geostatistical methods in the undulating rain-fed lands given the generally available hard information. By contrast, the variation in the gradually changing alluvial setting of Nueva Ecija was more reliably captured by the geostatistical approach as reflected by the validation statistics. Furthermore, indicator 

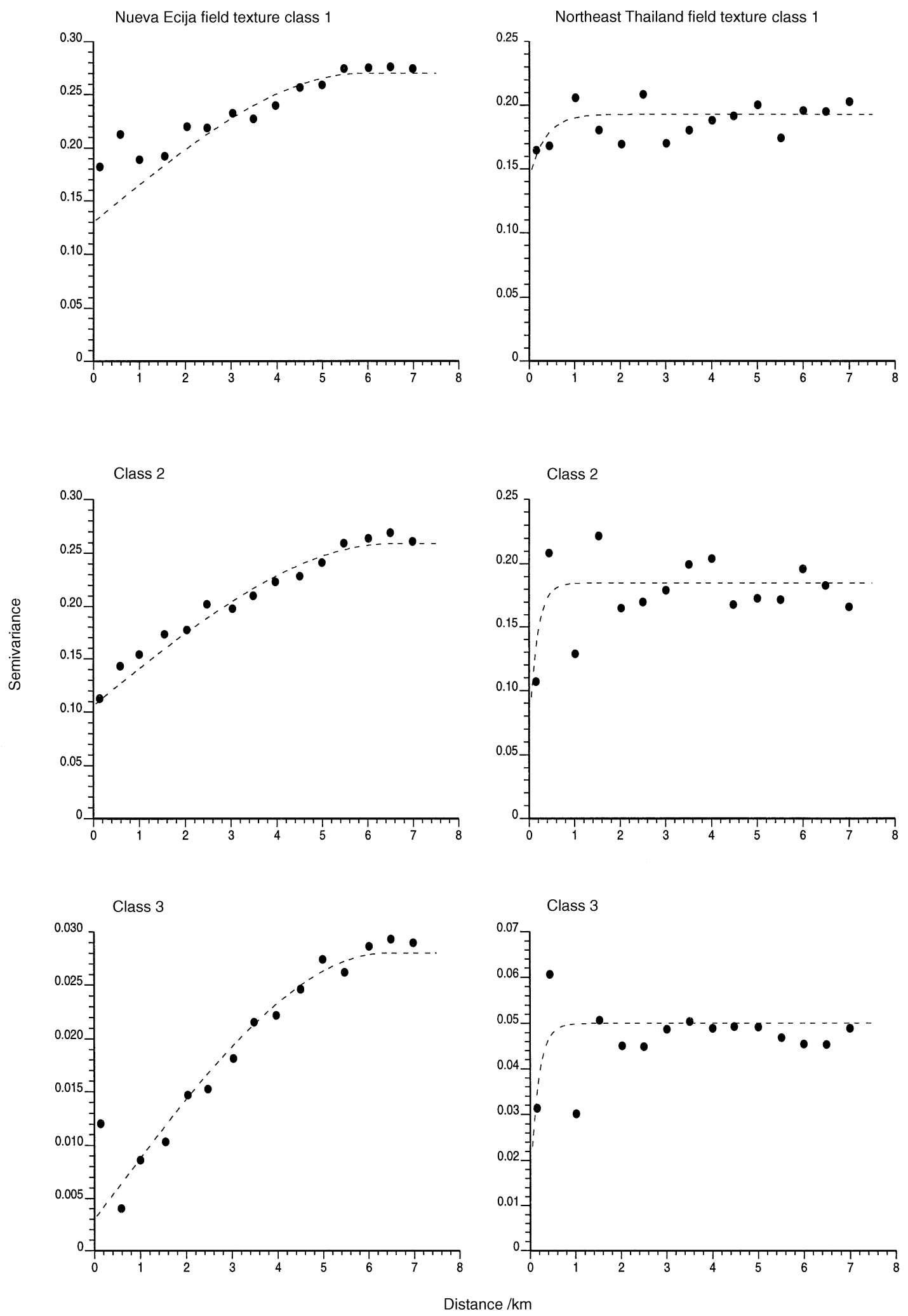

Figure 5 Experimental variograms of residuals and fitted models (dashed line) for soil texture classes in both areas. 
Table 6 Variogram parameters for the complete study area in Northeast Thailand (based on 278 samples)

\begin{tabular}{|c|c|c|c|c|c|c|c|}
\hline & $\mathrm{VM}_{1}{ }^{\mathrm{a}}$ & $c_{0}{ }^{\mathrm{b}}$ & $c_{1}{ }^{\mathrm{c}}$ & $r_{1} / \mathrm{m}^{\mathrm{d}}$ & $\mathrm{VM}_{2}{ }^{\mathrm{a}}$ & $c_{2}{ }^{\mathrm{e}}$ & $r_{2} / \mathrm{m}^{\mathrm{f}}$ \\
\hline \multicolumn{8}{|c|}{ Variograms } \\
\hline Clay & $\exp$ & 5.0 & 54.5 & 620 & - & - & - \\
\hline Silt & $\exp$ & 20.0 & 117.6 & 667 & - & - & - \\
\hline Sand & $\exp$ & 47.6 & 145.6 & 1411 & - & - & - \\
\hline \multicolumn{8}{|c|}{ Indicator variograms } \\
\hline Class 1 & $\exp$ & 0.120 & 0.175 & 136 & $\exp$ & 0.240 & 2038 \\
\hline Class 2 & nug & 0.235 & - & - & - & - & - \\
\hline Class 3 & $\exp$ & 0.061 & 0.166 & 467 & $\exp$ & 0.197 & 2628 \\
\hline \multicolumn{8}{|c|}{ Variograms of residuals } \\
\hline Class 1 & $\exp$ & 0.143 & 0.193 & 350 & - & - & - \\
\hline Class 2 & $\exp$ & 0.065 & 0.185 & 175 & - & - & - \\
\hline Class 3 & $\exp$ & 0.014 & 0.05 & 175 & - & - & - \\
\hline
\end{tabular}

${ }^{\mathrm{a}}$ Variogram models for the short-range $\left(\mathrm{VM}_{1}\right)$ and long-range structures $\left(\mathrm{VM}_{2}\right)$ : exp - exponential model, nug - pure nugget; ${ }^{\mathrm{b}}$ nugget variance; ${ }^{\mathrm{c}}$ sill of the short-range variance; ${ }^{\mathrm{d}}$ distance parameter of the short-range structure; ${ }^{\mathrm{e}}$ sill of the long-range variance; ${ }^{\mathrm{f}}$ distance parameter of the long-range structure.

\begin{tabular}{lcccccc}
\hline & $D$ & $\mathrm{SM}^{\mathrm{a}}-P$ & $\mathrm{TP}^{\mathrm{b}}-P$ & $\mathrm{OK}^{\mathrm{c}}-P$ & $\mathrm{IK}^{\mathrm{d}}-P$ & $\mathrm{SIK}^{\mathrm{e}}-P$ \\
\hline $\begin{array}{l}\text { Nueva Ecija } \\
\text { Class 1 }\end{array}$ & 0.44 & 0.94 & 0.45 & 0.28 & 0.44 & 0.44 \\
Class 2 & 0.28 & 0.06 & 0.29 & 0.46 & 0.28 & 0.28 \\
Class 3 & 0.28 & 0.00 & 0.26 & 0.26 & 0.28 & 0.28 \\
Northeast Thailand & & & & & & \\
Class 1 & 0.39 & 0.05 & 0.46 & 0.69 & 0.39 & 0.39 \\
Class 2 & 0.36 & 0.86 & 0.30 & 0.20 & 0.36 & 0.36 \\
Class 3 & 0.25 & 0.09 & 0.24 & 0.11 & 0.25 & 0.25 \\
\hline
\end{tabular}

Table 7 Relative proportions $(P)$ of texture classes for the interpolated maps, and the corresponding proportions for the field samples $(D)$

${ }^{\mathrm{a}}$ Soil map method; ${ }^{\mathrm{b}}$ Thiessen polygons; ${ }^{\mathrm{c}}$ ordinary kriging and GIS-based classification;

dindicator kriging using Soares's classification algorithm; 'indicator kriging with prior local means using Soares's classification algorithm.

kriging combined with Soares's classification algorithm reliably reproduced the distribution of soil texture classes in the survey data for both areas.

Soil maps are frequently used for agronomic planning or regional crop modelling in both environments, but they were not produced specifically for this purpose: the re-coded Nueva Ecija soil map tends to underestimate the clay and silt content. On the other hand, re-coding of the soil map overestimates the clay content for most of the land in Northeast Thailand.

Incorporating soft information did not improve mapping accuracy in Nueva Ecija, but the SIK map appears less fragmented than other maps that were generated by interpolation of field-estimated soil texture classes. The lack of improvement in accuracy when using SIK was expected given the small values of the $\hat{B}\left(s_{k}\right)$ coefficients, but we recommend the soil map as a source of complementary information if generating contiguous classes is of prime concern for regional land assessment and planning. In the undulating rain-fed lands of Northeast Thailand, knowledge of farmers and soil experts implicitly reflected the spatial variation of soil texture classes, and this soft information was incorporated in the geostatistical approach to improve mapping accuracy. Unlike in Nueva Ecija, the validation statistics strongly supported the integration of soft information. Soil texture classes followed a dendritic pattern, which reflects partly the local topography and drainage system. Considering only topography and drainage pattern as soft information would not be sufficient because correspondence between these features and soil 
Nueva Ecija

(a) Clay $/ \%$

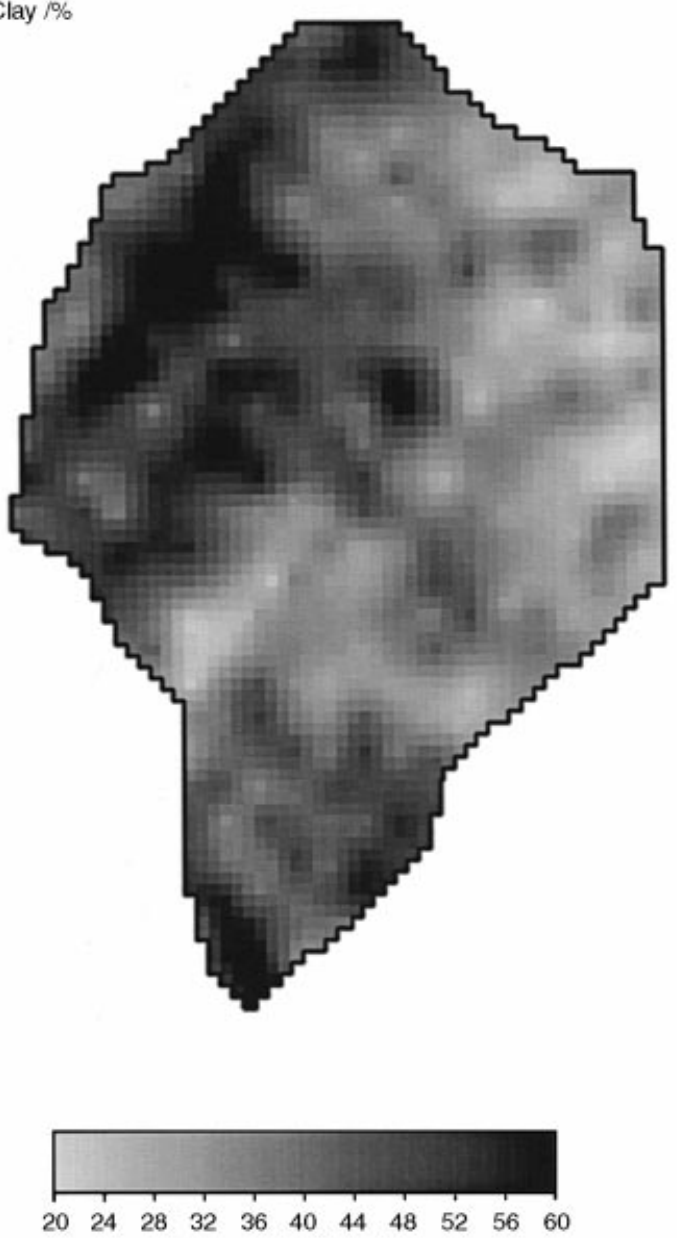

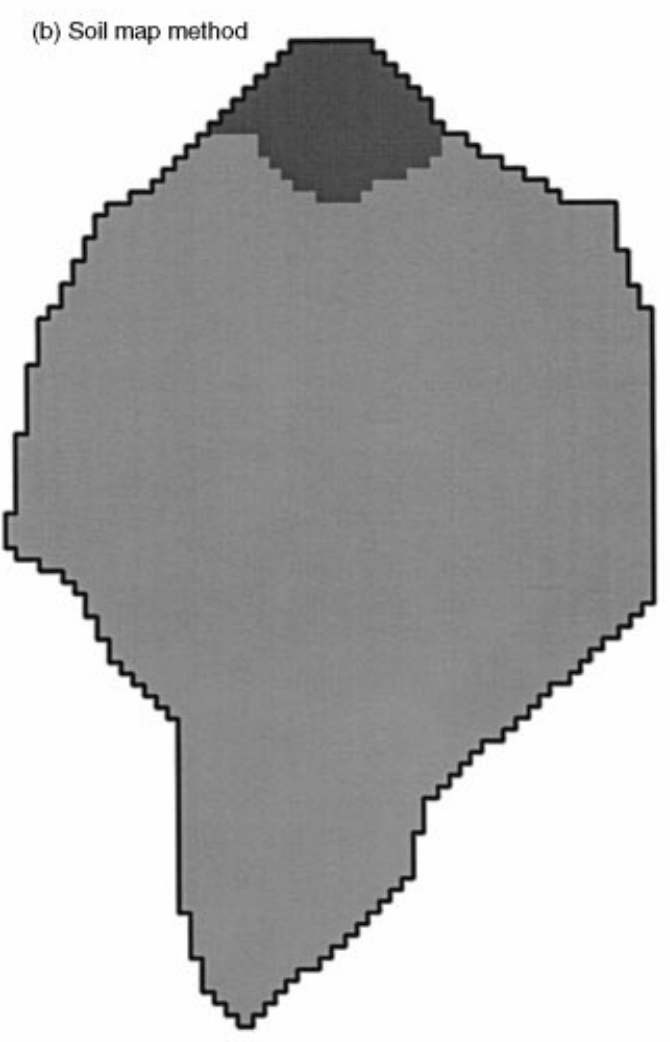

(c) Thiessen

polygons

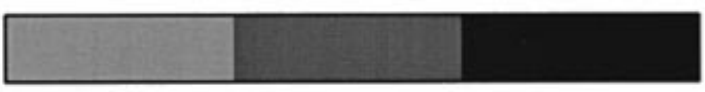

Field soil texture classes:

light

medium

heavy

Figure 6 Soil texture maps for the Nueva Ecija area. The clay content map (a) was created with ordinary kriging. Map (b) is the re-coded soil map for which soil texture classes of the representative unit profiles were assigned to the whole unit. The Thiessen polygons map (c) and the re-coded soil map were produced within the ARC/INFO GIS environment. 

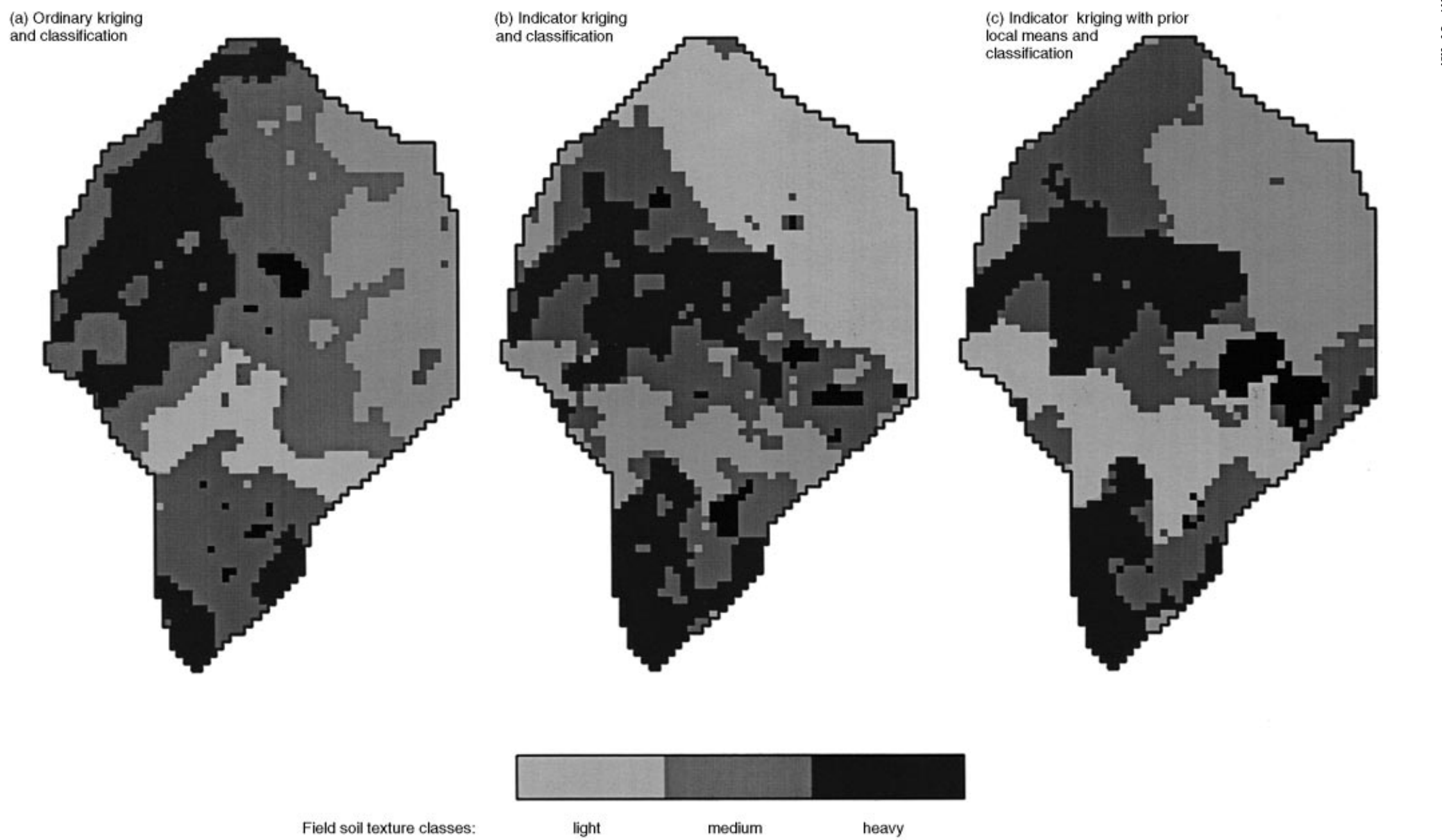

Figure 7 Soil texture maps for the Nueva Ecija area. The clay content map (Figure 6a) was used to generate map (a) by GIS classification. Maps (b) and (c) were generated by applying Soares's classification algorithm to probabilities of class occurrence, which were estimated using indicator kriging (b) and indicator kriging with local prior means (c). 
(a) Sand $/ \%$
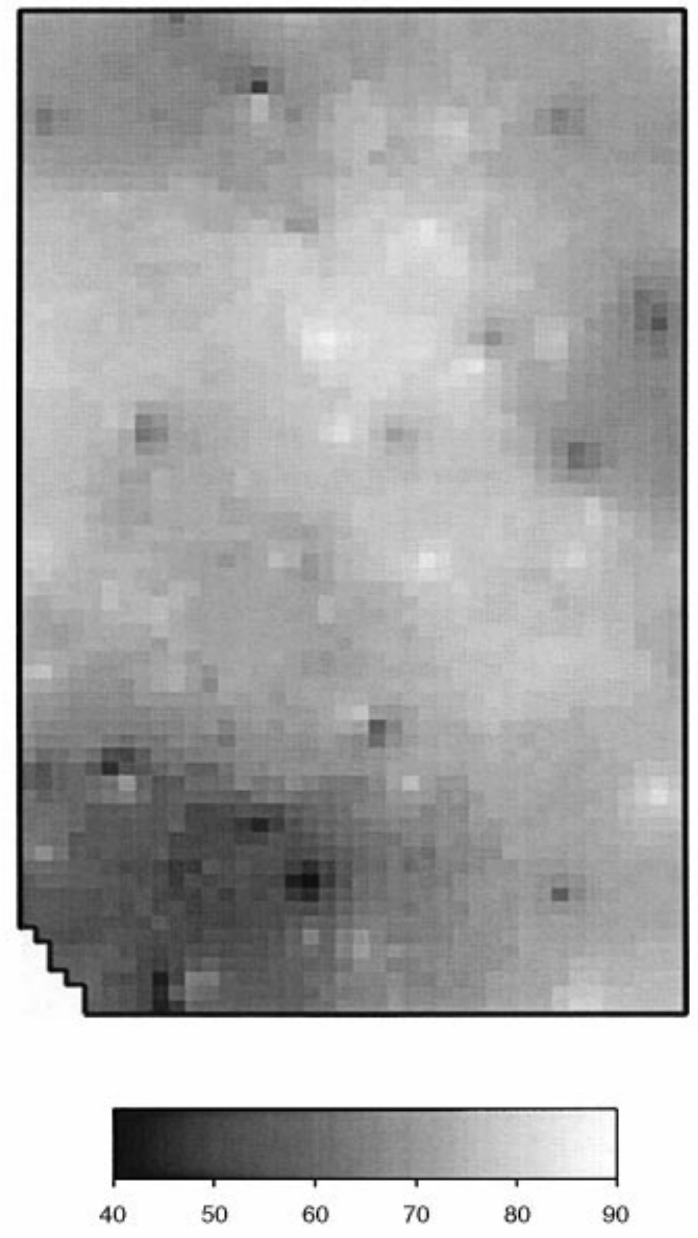

(b) Soil map method

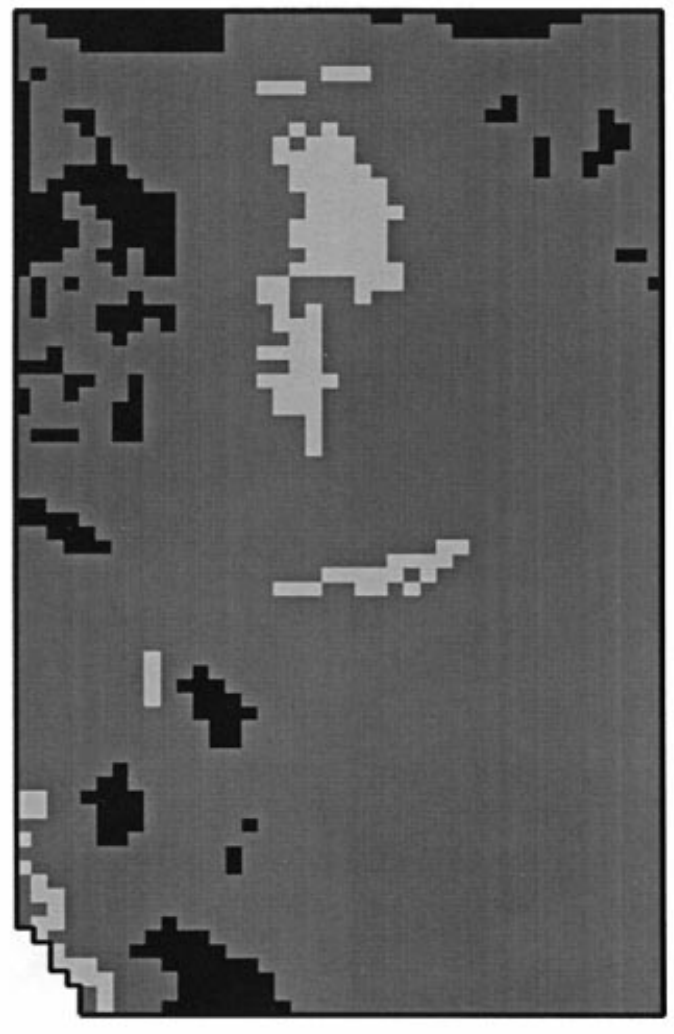

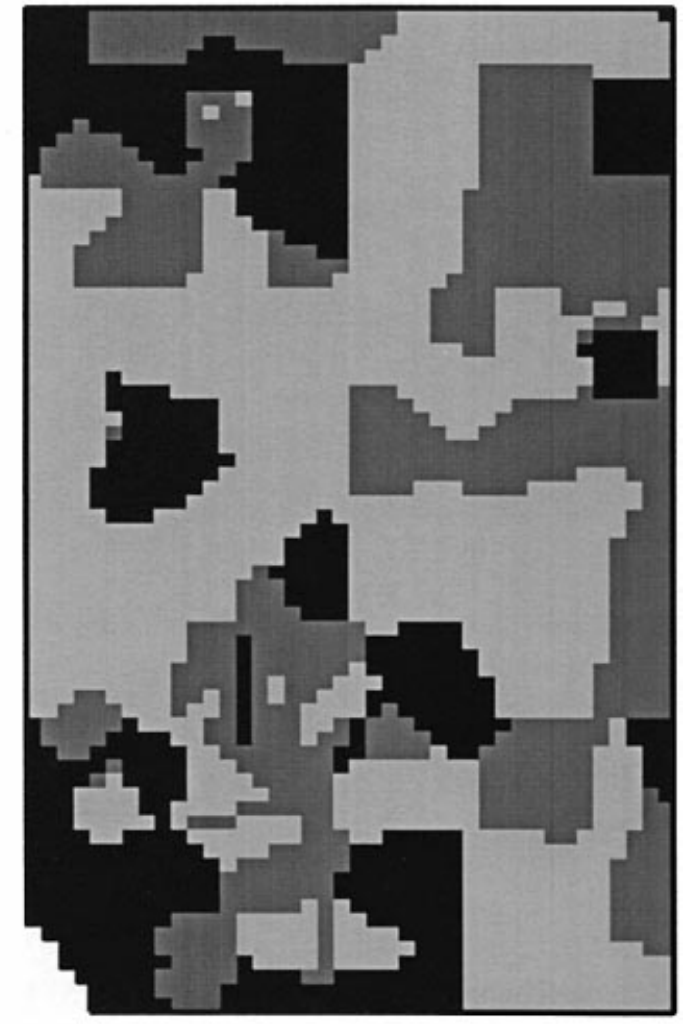

Field soil texture classes:

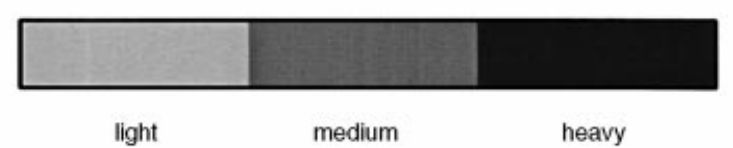

Figure 8 Soil texture maps for the Northeast Thailand subarea. The sand content map (a) was created with ordinary kriging. Map (b) is the re-coded soil map for which soil texture classes of the representative unit profiles were assigned to the whole unit. The Thiessen polygons map (c) and the re-coded soil map were produced within the ARC/INFO GIS environment. 
Table 8 Comparison of the different mapping approaches using the validation data set $(n=40)$, Nueva Ecija. Three criteria are considered: proportion of validation points that are correctly classified (PCC; class values and global value), the Kappa coefficient (here denoted $A$; class values and global value) and Jolayemi's $r$-statistic (global). There is no $r$-statistic available for the SM method, because the calculation involved division by zero

\begin{tabular}{lcccc}
\hline & Class 1 & Class 2 & Class 3 & Global \\
\hline Validation points $(n)$ & 18 & 11 & 11 & 40 \\
SM & & & & \\
$A$ & 0.83 & 0.09 & 0.00 & 0.40 \\
$r$ & -0.06 & -0.03 & 0.00 & -0.06 \\
TP $^{\mathrm{b}}$ PCC & & & & - \\
$A$ & 0.55 & 0.18 & 0.64 & 0.47 \\
$r$ & 0.19 & -0.13 & 0.50 & 0.19 \\
OK & & & & 0.15 \\
$A$ & & & & 0.64 \\
$r$ & 0.39 & 0.45 & 0.36 & 0.22 \\
IK $^{\mathrm{d}}$ PCC & 0.24 & 0.08 & & 0.13 \\
$A$ & & & & 0.55 \\
$r$ & 0.72 & 0.09 & 0.73 & 0.28 \\
SIK $^{\mathrm{e}}$ PCC & 0.26 & -0.15 & 0.54 & 0.19 \\
$A$ & & & & 0.53 \\
$r$ & 0.56 & 0.36 & 0.64 & 0.27 \\
\hline
\end{tabular}

${ }^{\mathrm{a}}$ Re-coding of the soil map; ${ }^{\mathrm{b}}$ Thiessen polygons; ${ }^{\mathrm{c}}$ ordinary kriging with GIS-based classification; dindicator kriging and classification using Soares's algorithm; 'indicator kriging with local prior means and classification using Soares's algorithm.

texture class distribution is only partial: the size of typical microcatchments in the area is often smaller than the extent of the contiguous areas of soil texture classes.

\section{Soil texture distribution}

The changes of particle size distribution arise from the alluvial nature of the Central Luzon Basin in Nueva Ecija. Variograms suggest gradual changes in the textural composition reflecting fluvial sedimentation patterns. Silt content remains fairly stable across the different soil map units, and spatial variation is isotropic, and we do not have an explanation for this. Clay and sand contents show a trend in direction $120^{\circ}$, which is perpendicular to the flow of the Talavera River system and coincides with the direction of the change in elevation over longer distances.

For the Northeast Thailand site, our results are not consistent with the general belief that soils of the Khorat Plateau are universally coarse textured (e.g. Ragland \& Boonpuckdee, 1988, pp. 1-20). The OK map (11\%), the TP map $(24 \%)$ and the IK and SIK maps (25\%) assign much land
Table 9 Comparison of the different mapping approaches using the validation data set $(n=36)$, Northeast Thailand. Three criteria are considered: proportion of validation points that are correctly classified (PCC; class values and global value), the Kappa coefficient (here denoted $A$; class values and global value) and Jolayemi's $r$-statistic (global)

\begin{tabular}{lcccc}
\hline & Class 1 & Class 2 & Class 3 & Global \\
\hline Validation points $(n)$ & 21 & 13 & 2 & 36 \\
SM $^{\mathrm{a}}$ PCC & 0.14 & 0.77 & 0.50 & 0.39 \\
$A$ & 1.00 & 0.01 & 0.12 & 0.09 \\
$r$ & & & & 0.06 \\
$\mathrm{TP}^{\mathrm{b}}$ PCC & 0.57 & 0.46 & 1.00 & 0.55 \\
$A$ & 0.40 & 0.22 & 0.21 & 0.27 \\
$r$ & & & & 0.13 \\
OK & & & & \\
$A$ & 0.71 & 0.15 & 0.50 & 0.50 \\
$r$ & 0.10 & -0.12 & 0.15 & 0.06 \\
$\mathrm{IK}^{\mathrm{d}}$ PCC & & & & 0.07 \\
$A$ & 0.48 & 0.69 & 0.00 & 0.53 \\
$r$ & 0.31 & 0.31 & -0.06 & 0.21 \\
SIK $^{\mathrm{e}}$ PCC & & & & 0.08 \\
$A$ & & & & \\
$r$ & 0.81 & 0.38 & 1.00 & 0.66 \\
\hline & 0.37 & 0.30 & 0.47 & 0.37 \\
& & & & 0.30 \\
\hline
\end{tabular}

${ }^{\mathrm{a}}$ Re-coding of the soil map; ${ }^{\mathrm{b}}$ Thiessen polygons; ${ }^{\mathrm{c}}$ ordinary kriging with GIS-based classification; ${ }^{\mathrm{d}}$ indicator kriging and classification using Soares's algorithm; indicator kriging with local prior means and classification using Soares's algorithm.

to soil texture class 3 (loam and heavier). A loamy soil is generally regarded as suitable for rice production. Only land with soil texture class 1 appears consistent with the general perception of sandy Northeast Thailand soils, but most mapping approaches allocate only about half of the area to this class $(\mathrm{OK}=69 \%, \mathrm{TP}=46 \%$, IK and $\mathrm{SIK}=39 \%)$. The reliance on soil maps may have contributed to the long-held views about soil texture in Northeast Thailand. In reality, soil texture in this region varies over short distances, and the soil maps do not depict these changes. Colluvio-alluvial processes over short distances and in situ soil development are as important factors for the distribution of soils in Northeast Thailand as is gradually changing Quaternary alluvial sedimentation.

\section{Conclusions}

1 Field estimates of soil texture are valuable surrogates for expensive and time-demanding laboratory measurements of soil texture classes.

2 Soil maps at scales of 1:100 000 to 1:125000 highlight the important soil texture classes in the region but do not allow for agronomic interpretations. Agronomic interpretations should 
NE Thailand

(a) Ordinary kriging
and classification

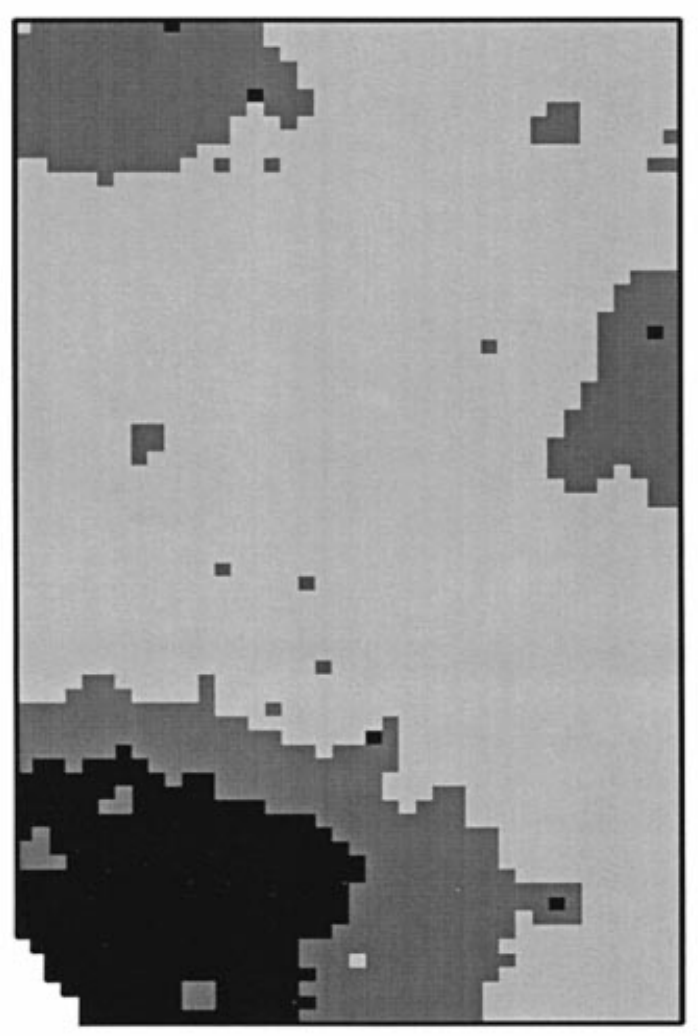

r kriging

and classification

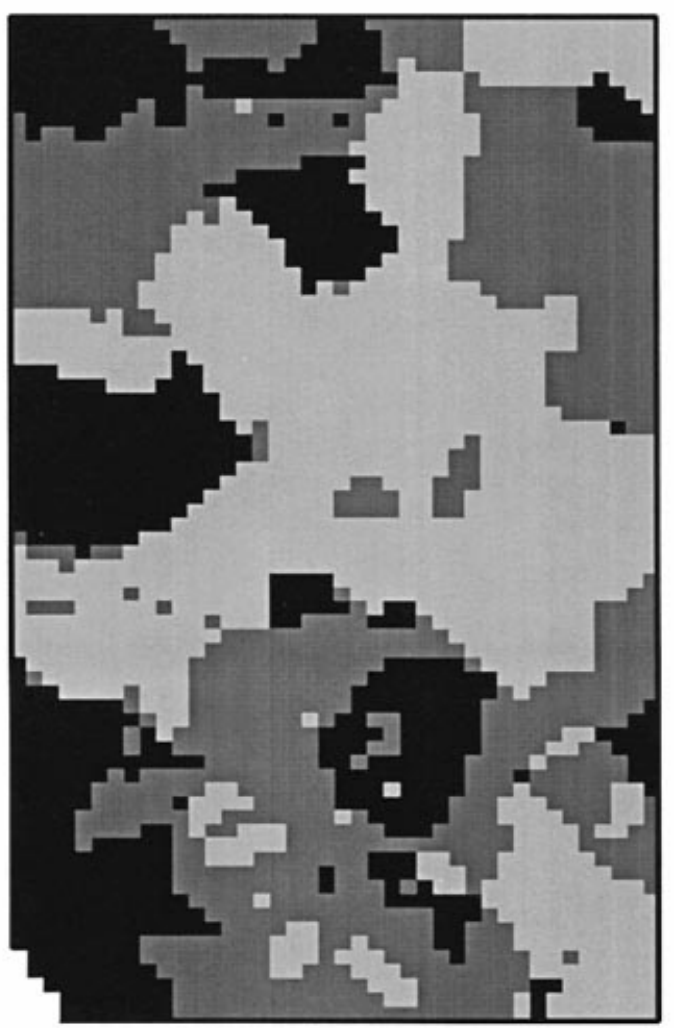

ng with prior local means and classification
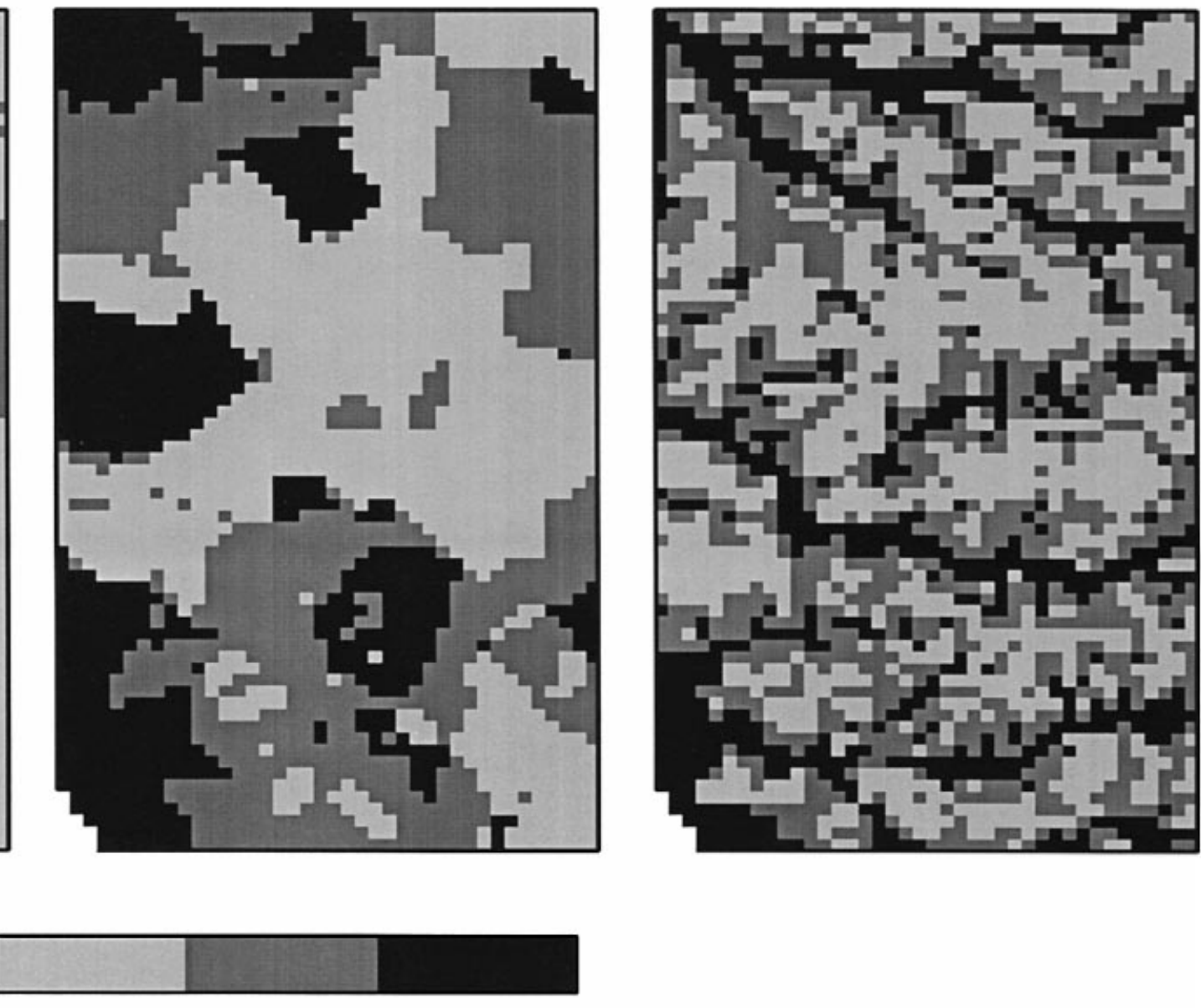

light

heavy

Figure 9 Soil texture maps for the Northeast Thailand subarea. The sand content map (Figure 8a) was used to generate map (a) by GIS classification. Maps (b) and (c) were generated by applying Soares's classification algorithm to probabilities of class occurrence, which were estimated using indicator kriging (b) and indicator kriging with local prior means (c). 
be based on maps generated by detailed soil surveys or on combining field texturing with TP, IK or SIK to produce refined maps.

3 Indicator kriging is technically feasible for mapping categorical data at regional scales in rain-fed and irrigated environments and in particular is better than simpler interpolation techniques where change is gradual. In heterogeneous environments such as Northeast Thailand simple interpolation is almost as good as the more complex indicator kriging.

4 Indicator kriging with prior local means is of particular value in the heterogeneous rain-fed lands where other interpolation techniques are not as good as the more complex SIK if complementary information is available for the interpolation.

5 The soft information used here is readily available in many regions or does not cost much to obtain. Therefore, instead of concentrating on additional sampling with greater density, SIK provides an interesting cheap alternative for updating or upgrading soil maps.

6 In Northeast Thailand, the integration of hard and soft information revealed visually that colluvio-alluvial processes act over short distances and in situ soil development is likely. Large-scale variation, as suggested by the variograms and SIK map, is not consistent with the widely held belief that Quaternary alluvial sedimentation was the sole process responsible for the distribution of soils in the region (see Moormann et al., 1964).

7 There is no ideal interpolation technique for the two ricegrowing areas. It is worth investigating whether other readily available information, such as elevation or local knowledge of Nueva Ecija, can improve prediction of soil texture classes.

\section{Acknowledgements}

Funding of the International Rice Research Institute (IRRI) and the University of Western Australia (UWA) made this study possible. We thank Mirasol Pampolino, Arlene Adviento, Wenceslao Larazo and Kongton Svichuen for their help in collecting the soil texture information and Arthur Conacher and Julie Delaney for reading our script, Simon Cook and Michelle Stuckey for all the ideas while the project evolved, and the editor and two anonymous referees for their guidance and suggestions. Most of all, we thank the farmers of Northeast Thailand for sharing with us their knowledge about the natural resources. We also acknowledge the support of the Bureau of Soils in Manila, the Soil Laboratory of the Department of Agriculture in Bangkok and the staff of the Rice Research Center in Ubon.

\section{References}

Alicante, M.M., Rosell, D.Z., Marfori, R.T. \& Hernandez, S. 1941. Soil Survey Report of the Nueva Ecija Province. Soil Report No 8, Department of Agriculture and Commerce, Manila.
Arrouays, D. 1987. Farmers' knowledge and soil survey: a multivariate treatment of agropedological data. Soil Survey and Land Evaluation, 7, 81-86.

Bárdossy, A. \& Lehmann, W. 1998. Spatial distribution of soil moisture in a small catchment. Part 1: Geostatistical analysis. Journal of Hydrology, 206, 1-15.

Bie, S.W. \& Beckett, P.H.T. 1971. Quality control in soil survey. II. The cost of soil survey. Journal of Soil Science, 22, 453-465.

Bishop, Y.M.M., Fienberg, S.E. \& Holland, P.W. 1975. Discrete Multivariate Analysis: Theory and Practice. MIT Press, Cambridge, MA.

Boucneau, G., Van Meirvenne, M., Thas, O. \& Hofman, G. 1998. Integrating properties of soil map delineations into ordinary kriging. European Journal of Soil Science, 49, 213-229.

Bregt, A.K., Stoorvogel, J.J., Bouma, J. \& Stein, A. 1992. Mapping ordinal data in soil survey: a Costa Rican example. Soil Science Society of America Journal, 56, 525-531.

Brus, D.J., De Gruijter, J.J., Marsman, B.A., Visschers, R., Bregt, A.K., Breeuwsma, A. \& Bouma, J. 1996. The performance of spatial interpolation methods and choropleth maps to estimate properties at points: a soil survey case study. Environmetrics, 7, 116.

Changprai, C., Chotimon, A., Thunduan, V., Thipsuwan, C., Lepananontha, J. \& Kittiyarak, S. 1971. Detailed Reconnaissance Soil Map of Ubon Ratchathani Province. Department of Land Development, Soil Survey Division, Bangkok.

Cohen, J. 1960. A coefficient of agreement for nominal scales. Educational and Psychological Measurement, 20, 37-46.

Deutsch, C.V. \& Journel, A.G. 1992. GSLIB. Geostatistical Software Library and User's Guide. Oxford University Press, New York.

ESRI 1991. ARC/INFO. Environmental Systems Research Institute Inc., Redlands, CA.

Finke, P.A., Wösten, J.H.M. \& Kroes, J.G. 1996. Comparing two approaches of characterizing soil map unit behavior in solute transport. Soil Science Society of America Journal, 60, 200-205.

Foody, G.M. 1994. Ordinal-level classification of sub-pixel tropical forest cover. Photogrammetric Engineering and Remote Sensing, 60, 61-65.

Goovaerts, P. 1994. Comparison of CoIK, IK, and MIK performances for modeling conditional probabilities of categorical variables. In: Geostatistics for the Next Century (ed. R. Dimitrakopoulos), pp. 18-29. Kluwer Academic Publishers, Dordrecht.

Goovaerts, P. 1997. Geostatistics for Natural Resources Evaluation. Oxford University Press, New York.

Goovaerts, P. \& Journel, A.G. 1995. Integrating soil map information in modelling the spatial variation of continuous soil properties. European Journal of Soil Science, 46, 397-414.

Heuvelink, G.B.M. \& Bierkens, M.F.P. 1992. Combining soil maps with interpolations from point observations to predict quantitative soil properties. Geoderma, 55, 1-15.

Hodgson, J.M., Hollis, J.M., Jones, R.J.A. \& Palmer, R.C. 1976. A comparison of field estimates and laboratory analyses of the silt and clay contents of some West Midland soils. Journal of Soil Science, 27, 411-419.

Jolayemi, E.T. 1990. On the measure of agreement between two raters. Biometrical Journal, 32, 87-93.

Journel, A. 1983. Non-parametric estimation of spatial distributions. 
Journal of the International Association for Mathematical Geology, 15, 445-468.

Journel, A.G. 1986. Constrained interpolation and qualitative information - the soft kriging approach. Mathematical Geology, 18, 269-286.

Kuntze, H., Röschmann, G. \& Schwerdtfeger, G. 1994. Bodenkunde. Verlag Eugen Ulmer, Stuttgart.

Landon, J.R. 1991. Booker Tropical Soil Manual. Longman, Harlow. Leenhardt, D., Voltz, M., Bornand, M. \& Webster, R. 1994. Evaluating soil maps for prediction of soil water properties. European Journal of Soil Science, 45, 293-301.

Moormann, F.R., Montrakun, S. \& Panichapong, S. 1964. Soils of Northeastern Thailand. A Key to their Identification and Survey. Department of Land Development, Soil Survey Division, Bangkok. Oberthür, T., Dobermann, A. \& Neue, H.U. 1996. How good is a reconnaissance soil map for agronomic purposes? Soil Use and Management, 12, 33-43.

Ragland, J. \& Boonpuckdee, L. 1988. Soil Fertility Management in
Northeast Thailand. Northeast Rainfed Agricultural Development Project, NERAD, Khon Kaen.

Sandor, J.A. \& Furbee, L. 1996. Indigenous knowledge and classification of soils in the Andes of Southern Peru. Soil Science Society of America Journal, 60, 1502-1512.

Soares, A. 1992. Geostatistical estimation of multi-phase structures. Mathematical Geology, 24, 149-160.

Soil Survey Staff 1951. Soil Survey Manual. US Department of Agriculture Handbook No 18, US Government Printing Office, Washington, DC.

Soil Survey Staff 1992. Keys to Soil Taxonomy. Soil Management Support Service Technical Monograph No 19, Pocahontas Press, Blacksburg, VA.

Voltz, M. \& Webster, R. 1990. A comparison of kriging, cubic splines and classification for predicting soil properties from sample information. Journal of Soil Science, 41, 473-490.

Webster, R. \& Oliver, M.A. 1990. Statistical Methods in Soil and Land Resource Survey. Oxford University Press, Oxford. 